A reprint from

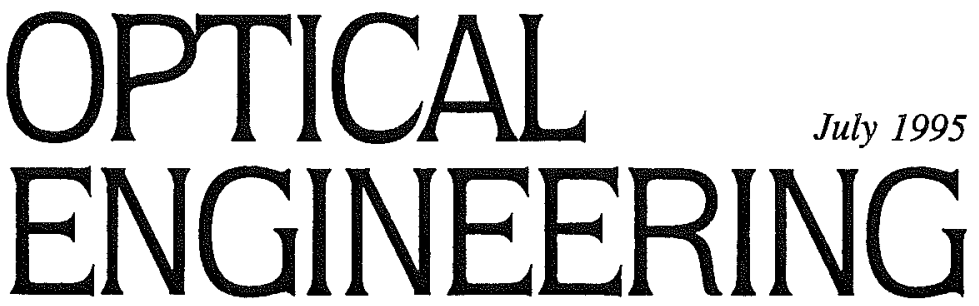

PROJECTION MOIRÉ: USING TENSOR CALCULUS FOR GENERAL

GEOMETRIES OF OPTICAL SETUPS

\author{
Philippe Tatasciore \\ Swiss Federal Institute of Technology \\ Institute of Lightweight Structures and Ropeways \\ Leonhardstrasse 25 \\ ETH-Zentrum, LEC \\ CH-8092 Zürich, Switzerland \\ E-mail: philippe@ils.mavt.ethz.ch \\ Erwin K. Hack \\ Swiss Federal Laboratories \\ for Materials Testing and Research \\ Section Electronics/Metrology \\ Überlandstrasse 129 \\ CH-8600 Dübendorf, Switzerland
}




\section{Projection moiré: using tensor calculus for general geometries of optical setups}

\section{Philippe Tatasciore}

Swiss Federal Institute of Technology

Institute of Lightweight Structures and

Ropeways

Leonhardstrasse 25

ETH-Zentrum, LEC

$\mathrm{CH}-8092$ Zürich, Switzerland

E-mail: philippe@ils.mavt.ethz.ch

Erwin K. Hack

Swiss Federal Laboratories for Materials Testing and Research

Section Electronics/Metrology

Überlandstrasse 129

$\mathrm{CH}-8600$ Dübendorf, Switzerland

\begin{abstract}
The projection moiré technique enables quantitative measurements of the shape of an opaque curved object surface in space. In contrast to the actual literature, where the interpretation of moire fringes is mostly limited to particular geometrical configurations, we present a strong mathematical tool that makes it possible to accurately analyze more general and complicated geometrical cases. We therefore introduce a mathematical model based on the calculation with projectors and use tensor calculus to deduce the general equations of projection moiré. Emphasis is put on relative moiré, which is used in most experiments, and on difference moiré, which is generally used to calibrate optical systems. The concept of a sensitivity vector, which comes essentially from holographic interferometry, is also introduced. Using a computer-based image processing system, a numerical experimental verification of the obtained theoretical tensor equations is performed. Simultaneously, we describe how to calibrate an optical setup and gain evidence of a few nonlinear effects. This shows which parameters of the setup are of importance and should be carefully controlled. Moreover, we treat some well-known special cases to demonstrate the agreement with the existing literature.
\end{abstract}

Subject terms: projection moiré; shadow moiré; projectors; tensors calculus; sensitivity vector; contouring; moiré fringes; object shape measurement.

Optical Engineering 34(7), 1887-1899 (July 1995).

\section{Introduction}

The shape of an opaque curved object surface in space can be quantitatively described using the projection moiré technique. ${ }^{1-13}$ Computer-based image processing systems have enabled a rapid development of the applications that use this technique, which as such is not new. By means of a light source $S$ (or projector), a grating $\hat{G}$ is projected onto the surface $G$ of an object* (Fig. 1). The observation of the projected grating $\hat{G}$ on the object through another grating $\widehat{G}$ from the point $R$ (or camera) enables seeing moiré fringes if the projections of the two gratings $\widehat{G}$ and $\hat{G}$ onto the object surface are similar. The whole information concerning the shape of the object surface is contained in these fringes. Assuming two sinusoidal gratings, the transmittance functions $\hat{T}$ of $\hat{G}$ and $\widehat{T}$ of $\widehat{G}$ are written

* Note that in this paper for simplicity most of the figures are drawn in two dimensions but in reality represent a 3-D situation.

Paper SWI-03 received Aug. 15, 1994; accepted for publication Oct. 7, 1994. (C) 1995 Society of Photo-Optical Instrumentation Engineers. 0091-3286/95/\$6.00.
$\hat{T}=\frac{1}{2}[1-\cos (2 \pi \hat{D})], \quad \widehat{T}=\frac{1}{2}[1-\cos (2 \pi \widehat{D})]$,

where $\hat{D}$ is the line order of grating $\hat{G}$ and $\widehat{D}$ that of $\bar{G}$. Maximal transmittance (i.e., a white line) is reached for $\hat{T}=1$ or $\widehat{T}=1$, respectively, and no transmittance (i.e., a black line) for $\hat{T}=0$ or $\hat{T}=0$. The intensity distribution $I_{P}$ over the object shape depends on the intensity $I_{S}$ of the projector and the transmittance $\hat{T}$ of the grating $\hat{G}$. In a similar way, the intensity $I_{R}$ received by the camera depends on the intensity $I_{P}$ over the object and the transmittance $\overparen{T}$ of the grating $\bar{G}$. Assuming a uniform intensity, we have $I_{P}=I_{S} \hat{T}=I_{S}[1-\cos (2 \pi \hat{D})] / 2$ and

$$
\begin{aligned}
I_{R}=I_{P} \widehat{T}=I_{S} \hat{T} \widehat{T}= & \frac{I_{S}}{4}[1-\cos (2 \pi \widehat{D})-\cos (2 \pi \hat{D})] \\
& +\frac{I_{S}}{8} \cos \left(2 \pi D_{m}\right)+\frac{I_{S}}{8} \cos \left(2 \pi D_{M}\right) .
\end{aligned}
$$

In Eq. (2) for the intensity $I_{R}$, the term before the last term represents an invisible high-frequency moiré with fringe order $D_{m}=\widehat{D}+\hat{D}$ and the last term a visible low-frequency 


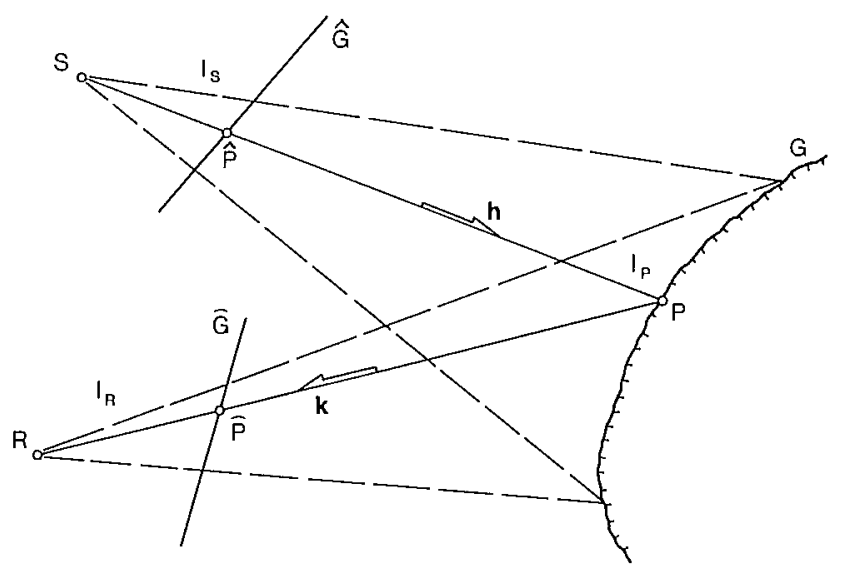

Fig. 1 Principle of projection moiré.

moiré with fringe order $D_{M}=\widehat{D}-\hat{D}$ (Fig. 2). Here we study only the visible moire $D_{M}$, which contains the needed information on the shape of the object surface.

\section{Optical Model}

The optical system of the camera (like that of the projector) can be described in the ideal case by the following geometrical relations (Fig. 3)

$\frac{1}{f}=\frac{1}{s}+\frac{1}{s^{*}}=\frac{1}{L_{e}}-\frac{1}{L_{e}^{*}}$

where $f$ is the focal length; $s$ and $s *$ are, respectively, the distances from the principal points $H$ and $H^{*}$ to the "object" and "image" points $K_{m}$ and $K_{m}^{*}$; and $L_{e}$ and $L_{e}^{*}$ are, respectively, the distances from the principal points $H$ and $H^{*}$ to the collineation centers $R$ and $R^{*}$. For the camera, the projection centers $R$ and $R^{*}$ are, respectively, located in the entrance pupil (aperture stop) and in the exit pupil. ${ }^{1}$ With $\xi / \xi^{*}=(s-f) / f$, we can define in this optical model a virtual collinear image $K_{v}$ on the object side associated to the point $K$ and its image $K^{*}$ such that

$\xi_{v}=\xi^{*}, \quad \frac{\xi_{v}}{s_{v}-L_{e}}=\frac{\xi}{s-L_{e}} \Rightarrow s_{\nu}=f\left(\frac{s-L_{e}}{s-f}\right)+L_{e}$,

where $\xi, \xi^{*}$, and $\xi_{v}$ are, respectively, positive distances in the object, image, and virtual image planes of the optical system.

\section{Line Order of the Camera Grating}

The line order $\bar{D}$ of the camera grating is first defined on the camera grid-plane in point $\widehat{P}$ (Fig. 4) and then extended in the space $\mathscr{R}^{3}$ by central projection relatively to the collineation center $R$ (see Fig. 7 in Sec. 4). The value of the dimensionless scalar $\widehat{D} \in \mathscr{R}$ is the same on the straight line passing through the points $R, \widehat{P}, P$, and $K$. In case of a grating with equidistant straight lines, the expression for $\overparen{D}$ is written

$\widehat{D}=\widehat{D}(\widehat{\mathbf{r}})=\frac{1}{\bar{\lambda}} \widehat{\mathbf{g}} \cdot \widehat{\mathbf{N}}\left(\widehat{\mathbf{r}}-\widehat{\mathbf{r}}_{c}\right)$

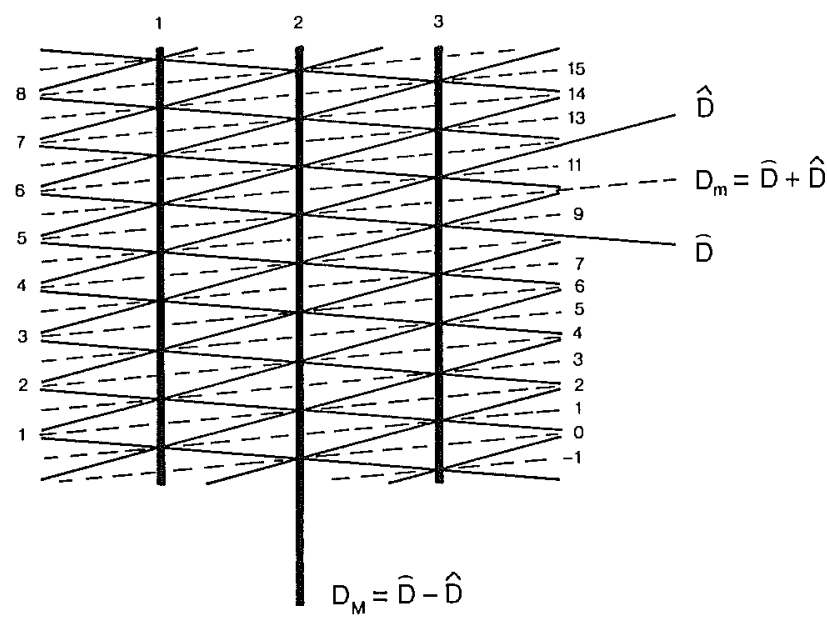

Fig. 2 Formation of moiré fringes.

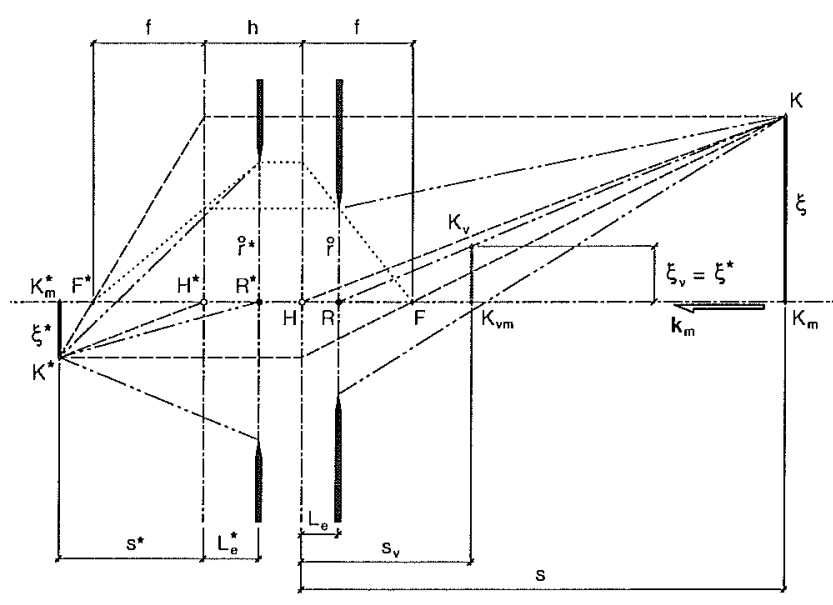

Fig. 3 Model of the optical system.

where the sign - indicates a scalar product of two vectors. The tensor $\mathbf{N}=\mathbf{I}-\overline{\mathbf{n}} \otimes \overline{\mathbf{n}}$ is a normal projector, where the sign $\otimes$ describes a tensor product (or dyadic product) of two vectors, and the vector $\mathbf{n}$ is a unit vector normal to the grid plane. The projector $\widehat{N}$ projects along the direction $\widehat{\mathbf{n}}$ onto the grid plane normal to $\widehat{\mathbf{n}}$ (Fig. 5) and the tensor $\mathbf{I}$ represents $^{14,15}$ the identity in the space $\mathscr{R}^{3}$. The constant scalar value $\bar{\lambda}$ represents the line spacing between two neighboring lines on the camera grid plane; the characteristic unit vector $\widehat{\mathbf{g}} \equiv \widehat{\mathbf{N}} \mathbf{g}$ is situated in the camera grid plane $(\widehat{\mathbf{g}} \perp \widehat{\mathbf{n}})$ and is perpendicular to the grating lines. The vector $\widehat{\mathbf{r}}$ can be interpreted as a vector variable giving the position of point $\widehat{P}$ in the camera grid plane, where $\overrightarrow{\mathbf{r}}_{c}$ represents the vector coordinate of some reference point on the grid plane such that $\widehat{D}\left(\mathbf{r}_{c}\right)=0$

\section{Line Order of the Projector Grating}

The line order $\hat{D}$ of the projector grating is also first defined on the projector grid plane in point $\hat{P}$ (Fig. 6) and then extended in the space $\mathscr{R}^{3}$ by central projection relatively to the collineation center $S$ (Fig. 7). The value of the dimensionless scalar $\hat{D} \in \mathscr{R}$ is the same on the straight line passing through 


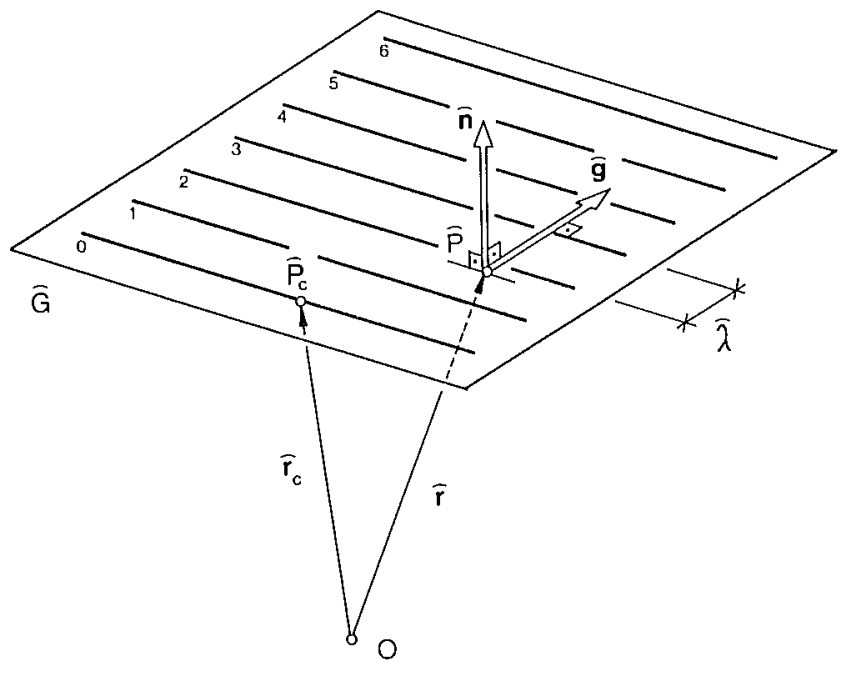

Fig. 4 Line order of the camera grating on the grid plane.

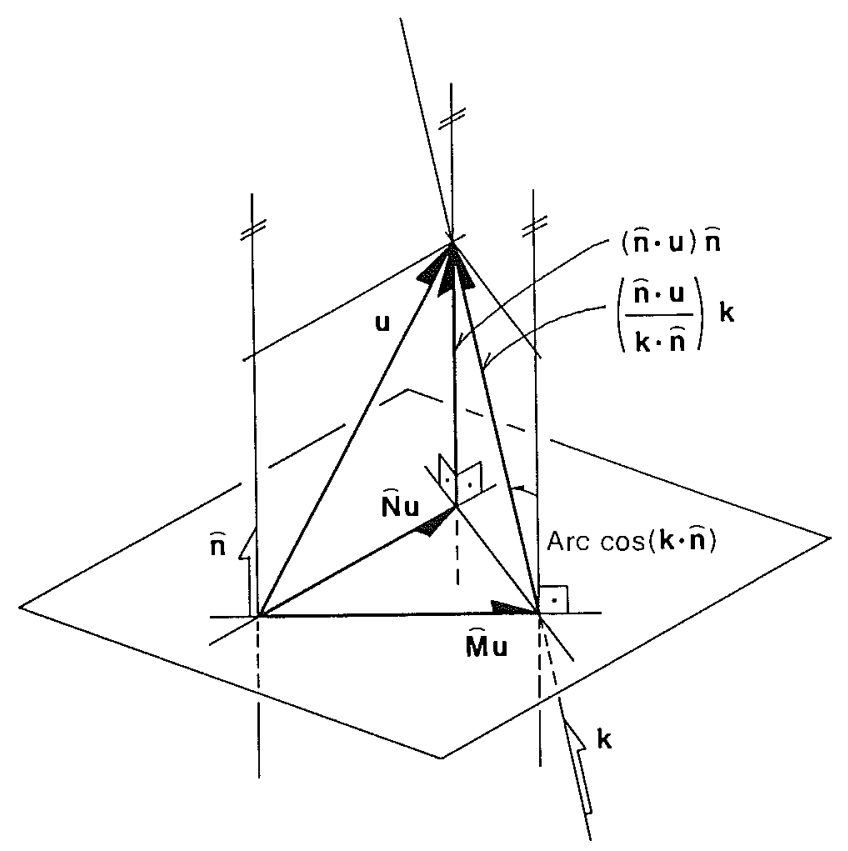

Fig. 5 Oblique and normal projections.

the points $S, \hat{P}, P$, and $Q$. In the case of a grating with equidistant straight lines, the expression for $\hat{D}$ is written

$\hat{D}=\hat{D}(\hat{\mathbf{r}})=\frac{1}{\hat{\lambda}} \hat{\mathbf{g}} \cdot \hat{\mathbf{N}}\left(\hat{\mathbf{r}}-\hat{\mathbf{r}}_{c}\right)-\Psi, \quad$ with $\quad \Psi=\frac{1}{\hat{\lambda}} \hat{\mathbf{g}} \cdot \Delta \hat{\mathbf{r}}_{c}$,

$\Delta \hat{\mathbf{r}}_{c} \equiv \hat{\mathbf{N}} \Delta \hat{\mathbf{r}}_{c}=\Psi \hat{\lambda} \hat{\mathbf{g}}$

The tensor $\hat{\mathbf{N}}=\mathbf{I}-\hat{\mathbf{n}} \otimes \hat{\mathbf{n}}$ is a normal projector and the vector $\hat{\mathbf{n}}$ is a unit vector normal to the grid plane; $\hat{\mathbf{N}}$ projects along the direction $\hat{\mathbf{n}}$ onto the grid plane. The constant scalar value $\hat{\lambda}$ represents the line spacing between two neighboring lines on the projector grid plane; the characteristic unit vector $\hat{\mathbf{g}}=\hat{\mathbf{N}} \hat{\mathbf{g}}$ is situated in the projector grid plane $(\hat{\mathbf{g}} \perp \hat{\mathbf{n}})$ and is perpendicular to the grating lines. The vector $\hat{\mathbf{r}}$ is a vector

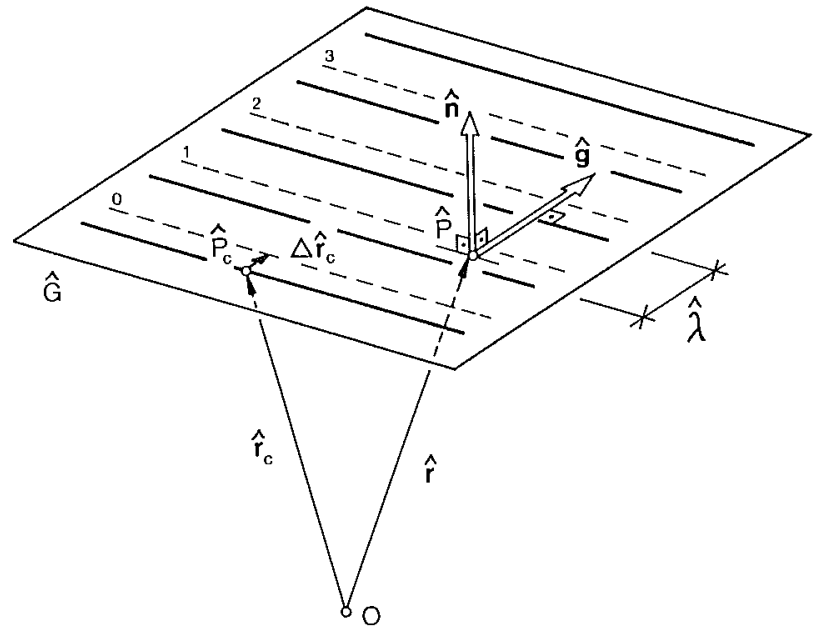

Fig. 6 Line order of the projector grating on the grid plane.

variable giving the position of point $\hat{P}$ in the projector grid plane, with $\hat{\mathbf{r}}_{c}$ representing the vector coordinate of some reference point on the grid plane such that $\hat{D}\left(\hat{\mathbf{r}}_{c}\right)=-\Psi$. The scalar increment $\Psi$ plays an important role in the phaseshifting method to get a phase image ${ }^{2,16}$ and the vector increment $\Delta \hat{\mathbf{r}}_{c}=\Psi \hat{\lambda} \hat{\mathbf{g}}$ describes a uniform in-plane translation of the grating on the grid plane, the translation being in the direction of $\hat{\mathbf{g}}$ for $\Psi$ positive.

\section{Moiré Fringe Order}

Considering two fixed collineation centers $R$ and $S$ with, respectively, two fixed grating $\widehat{G}$ and $\hat{G}$ in space, the vector variables $\widehat{\mathbf{r}}$ and $\hat{\mathbf{r}}$ are not independent (Fig. 7). Both are functions of the vector coordinate $\mathbf{r}$ of some point $P$ in the space $\mathscr{R}^{3}$, which means $\mathbf{r} \rightarrow \widehat{\mathbf{r}}=\widehat{\mathbf{r}}(\mathbf{r})$ and $\mathbf{r} \rightarrow \hat{\mathbf{r}}=\hat{\mathbf{r}}(\mathbf{r})$. The moiré fringe order $D_{M}$ in point $P$ is then written as follows:

$$
\begin{aligned}
D_{M}= & D_{M}(\mathbf{r})=D_{M}(\widehat{\mathbf{r}}, \hat{\mathbf{r}})=\bar{D}-\hat{D} \\
& =\frac{1}{\widehat{\lambda}} \widehat{\mathbf{g}} \cdot \widehat{\mathbf{N}}\left(\widehat{\mathbf{r}}-\widehat{\mathbf{r}}_{c}\right)-\frac{1}{\hat{\lambda}} \hat{\mathbf{g}} \cdot \hat{\mathbf{N}}\left(\hat{\mathbf{r}}-\hat{\mathbf{r}}_{c}\right)+\Psi .
\end{aligned}
$$

Note that a moiré surface in the space $\mathscr{R}^{3}$ can be described by a set of vectors $\mathbf{r}=\mathbf{r}_{M}$ for which the moiré fringe order $D_{M}$ is constant $(\phi=$ constant $)$, which means $\mathbf{r}_{M} \rightarrow$ $D_{M}\left(\mathbf{r}_{M}\right)=\varnothing$. On another hand, we can choose for our purposes the point $P$ on the object surface $G$ and look at the behavior of $D_{M}$. This is useful for the study of the object shape. Then, for a given fixed object surface $G$, the function $D_{M}$ can also by definition be extended in the space $\mathscr{R}^{3}$ by central projection relatively to the collineation center $R$, its value being the same on the straight line passing through $R$, $\overparen{P}, P$, and $K$. For another point $P_{0}$, e.g., on the object surface, we have

$$
\begin{aligned}
D_{M 0} & =D_{M}\left(\mathbf{r}_{0}\right)=D_{M}\left(\widehat{\mathbf{r}}_{0}, \hat{\mathbf{r}}_{0}\right)=\widehat{D}_{0}-\hat{D}_{0} \\
& =\frac{1}{\hat{\lambda}} \widehat{\mathbf{g}} \cdot \widehat{\mathbf{N}}\left(\widehat{\mathbf{r}}_{0}-\widehat{\mathbf{r}}_{c}\right)-\frac{1}{\hat{\lambda}} \hat{\mathbf{g}} \cdot \hat{\mathbf{N}}\left(\hat{\mathbf{r}}_{0}-\hat{\mathbf{r}}_{c}\right)+\Psi_{0},
\end{aligned}
$$




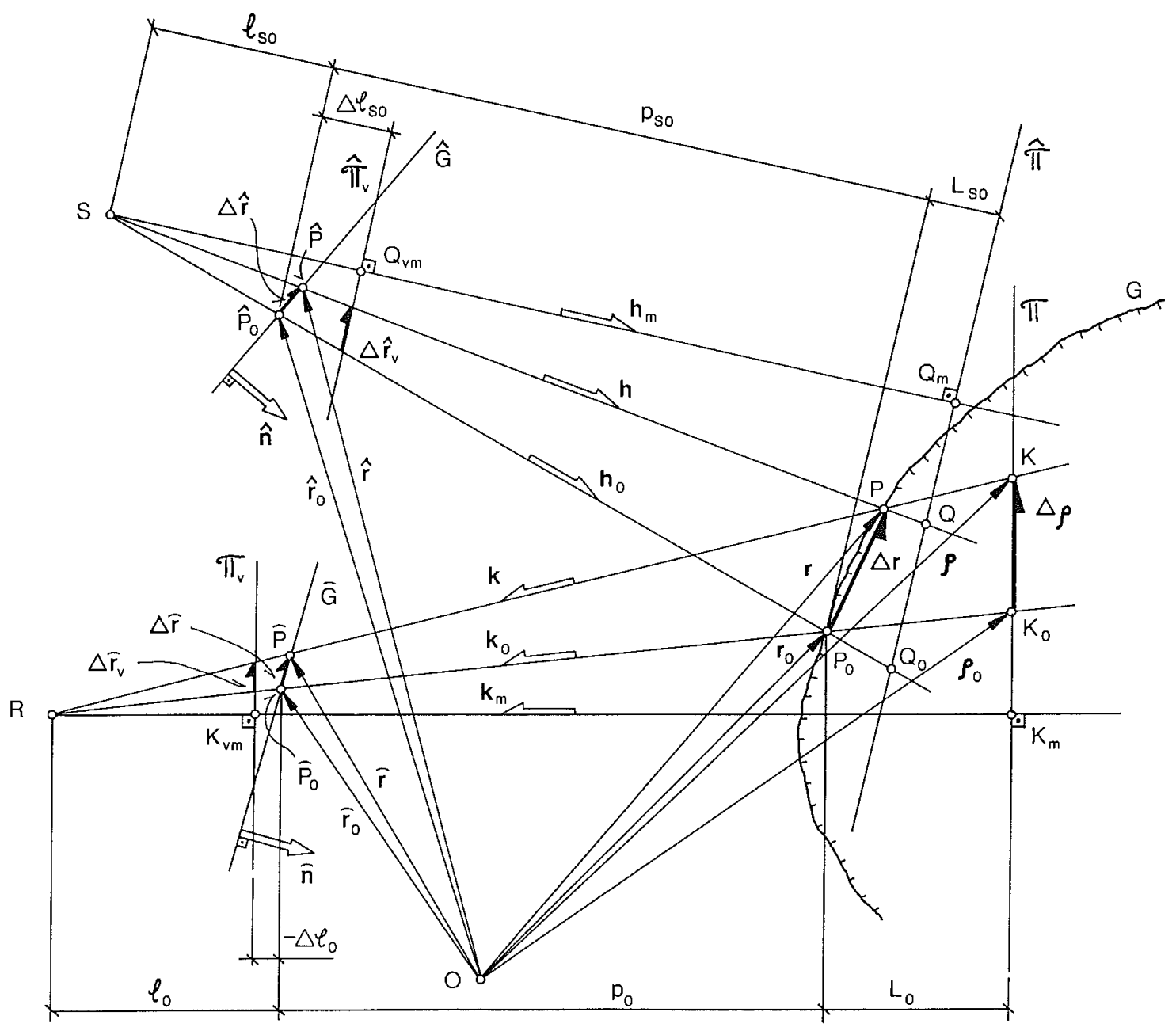

Fig. 7 Model of a general geometrical moiré setup.

where $\widehat{D}_{0}=\widehat{D}\left(\mathbf{r}_{0}\right)=\widehat{\mathbf{g}} \cdot \widehat{\mathbf{N}}\left(\mathbf{r}_{0}-\widehat{\mathbf{r}}_{c}\right) / \widehat{\lambda}$ and $\hat{D} 0=\hat{D}\left(\hat{\mathbf{r}}_{0}\right)=$ $\left[\hat{\mathbf{g}} \cdot \hat{\mathbf{N}}\left(\hat{\mathbf{r}}_{0}-\hat{\mathbf{r}}_{c}\right) / \hat{\lambda}\right]-\Psi_{0}$ are, respectively, the line order of the camera grating in $P_{0}$ and that of the projector grating in $\hat{P}_{0}$. In most cases, $\Psi$ and $\Psi_{0}$ are the same. We use the value $D_{M 0}$ later as a reference for the other values of $D_{M}$.

\section{General Expression of Relative Moiré}

We can now define a relative moiré value $\Delta D_{M}$ in some point $P$ in the space $\mathscr{R}^{3}$ by taking the value of the moire fringe order in a point $P_{0}$ as reference (Fig. 7). We write for $\Psi=\Psi_{0}$,

$$
\begin{aligned}
\Delta D_{M} & =D_{M}-D_{M 0}=D_{M}(\mathbf{r})-D_{M}\left(\mathbf{r}_{0}\right) \\
& =\left(\widehat{D}-\widehat{D}_{0}\right)-\left(\hat{D}-\hat{D}_{0}\right) \\
& =\frac{1}{\lambda} \widehat{\mathbf{g}} \cdot \widehat{\mathbf{N}}\left(\widehat{\mathbf{r}}-\widehat{\mathbf{r}}_{0}\right)-\frac{1}{\hat{\lambda}} \hat{\mathbf{g}} \cdot \hat{\mathbf{N}}\left(\hat{\mathbf{r}}-\hat{\mathbf{r}}_{0}\right) \\
& =\frac{1}{\frac{1}{\lambda}} \overline{\mathbf{g}} \cdot \Delta \overrightarrow{\mathbf{r}}-\frac{1}{\hat{\lambda}} \hat{\mathbf{g}} \cdot \Delta \hat{\mathbf{r}},
\end{aligned}
$$

where $\Delta \mathbf{r} \equiv \widehat{\mathbf{N}}\left(\widehat{\mathbf{r}}-\widehat{\mathbf{r}}_{0}\right)$ and $\Delta \hat{\mathbf{r}} \equiv \hat{\mathbf{N}}\left(\hat{\mathbf{r}}-\hat{\mathbf{r}}_{0}\right)$ are, respectively, the vector increments in the camera and projector grid planes. The dimensionless scalar $\Delta D_{M}$ is simply called relative moiré in point $P$. Because $D_{M 0}=\not$, the moiré surfaces are still described by a set of vectors $\mathbf{r}=\mathbf{r}_{M}$ for which $\Delta D_{M}=\phi$. Equation (9) is general and is valid for all geometrical configurations of the optical setup. For a given fixed object surface $G$, the relative moire $\Delta D_{M}$ can be extended in space like the moiré fringe order $D_{M}$.

To make Eq. (9) explicit, it is necessary to introduce some tensor relations called affine connections making the bridge among the vectors $\Delta \mathbf{r}, \Delta \widehat{\mathbf{r}}, \Delta \hat{\mathbf{r}}, \Delta \widehat{\mathbf{r}}_{v}, \Delta \hat{\mathbf{r}}_{v}, \Delta \boldsymbol{\rho}$, and $\Delta \boldsymbol{\rho}_{v}$. These affine connections involve normal and oblique projectors in $\mathscr{R}^{3}$, also called affinors. ${ }^{14,15}$ Figure 7 shows that $\Delta \mathbf{r}=\mathbf{r}-\mathbf{r}_{0}$ is the vector from $P_{0}$ to $P$, that $\Delta \widehat{\mathbf{r}}_{v}$ and $\Delta \hat{\mathbf{r}}_{v}$ are, respectively, the collinear images of $\Delta \overrightarrow{\mathbf{r}}$ and $\Delta \hat{\mathbf{r}}$ in the virtual image planes of the camera and the projector, and that $\Delta \rho$ is the collinear image of $\Delta \mathbf{r}$ in the object plane of the optical system of the camera. With the unit vectors $\mathbf{k}, \mathbf{k}_{m}, \mathbf{h}$, and $\mathbf{h}_{m}$, we first define the two normal projectors $\mathbf{K}_{m}=\mathbf{I}-\mathbf{k}_{m} \otimes \mathbf{k}_{m}$ and $\mathbf{H}_{m}=\mathbf{I}-\mathbf{h}_{m} \otimes \mathbf{h}_{m}$, with $\mathbf{K}_{m}$ projecting onto a plane perpendicular to the optical axis of the camera, which means along the direction $\mathbf{k}_{n}$ onto a plane normal to $\mathbf{k}_{m}$, and with $\mathbf{H}_{m}$ projecting onto a plane perpendicular to the optical axis of the projector, which means along $\mathbf{h}_{m}$ onto a plane normal to $\mathbf{h}_{m}$. Both normal projectors $\mathbf{K}_{m}$ and $\mathbf{H}_{m}$ are symmetric. We then define the following oblique projectors (not symmetric): 


$$
\begin{aligned}
& \widehat{\mathbf{M}}=\mathbf{I}-\frac{\mathbf{k} \otimes \hat{\mathbf{n}}}{\mathbf{k} \cdot \hat{\mathbf{n}}}, \quad \hat{\mathbf{M}}=\mathbf{I}-\frac{\mathbf{h} \otimes \hat{\mathbf{n}}}{\mathbf{h} \cdot \hat{\mathbf{n}}}, \quad \widehat{\mathbf{M}}_{m}=\mathbf{I}-\frac{\mathbf{k} \otimes \mathbf{k}_{m}}{\mathbf{k} \cdot \mathbf{k}_{m}}, \\
& \hat{\mathbf{M}}_{m}=\mathbf{I}-\frac{\mathbf{h} \otimes \mathbf{h}_{m}}{\mathbf{h} \cdot \mathbf{h}_{m}}, \quad \mathbf{M}_{m}=\mathbf{I}-\frac{\mathbf{h}_{m} \otimes \mathbf{k}_{m}}{\mathbf{h}_{m} \cdot \mathbf{k}_{m}},
\end{aligned}
$$

with $\overline{\mathbf{M}}$ projecting along $\mathbf{k}$ onto a plane normal to $\widehat{\mathbf{n}}$ (Fig. 5), with $\hat{\mathbf{M}}$ projecting along $\mathbf{h}$ onto a plane normal to $\hat{\mathbf{n}}$, with $\widehat{\mathbf{M}}_{m}$ projecting along $\mathbf{k}$ onto a plane normal to $\mathbf{k}_{m}$, with $\hat{\mathbf{M}}_{m}$ projecting along $\mathbf{h}$ onto a plane normal to $\mathbf{h}_{m}$, and with $\mathbf{M}_{m}$ projecting along $\mathbf{h}_{m}$ onto a plane normal to $\mathbf{k}_{m}$. Note that this description is valid only if the vector is applied "on the right", of the oblique projector (i.e., $\widehat{\mathbf{M}} \Delta \mathbf{r}$ ). The two directions $\mathbf{k}$ and $\mathbf{h}$ are commonly called observing and illuminating direction. With the preceding definitions, the affine connections are written as follows

$$
\begin{aligned}
& \Delta \widehat{\mathbf{r}} \equiv \widehat{\mathbf{N}} \Delta \widehat{\mathbf{r}}=\frac{\ell_{0}}{\ell_{0}+p_{0}} \widehat{\mathbf{M}} \Delta \mathbf{r}=\frac{\ell_{0}}{\ell_{0}+p_{0}+L_{0}} \overline{\mathbf{M}} \Delta \boldsymbol{\rho} \\
& =\frac{\ell_{0}}{\ell_{0}+\Delta \ell_{0}} \widehat{\mathbf{M}} \Delta \widehat{\mathbf{r}}_{v}=\frac{\ell_{0}}{\ell_{0}+\Delta \ell_{0}} \widehat{\mathbf{M}} \Delta \boldsymbol{\rho}_{v}, \\
& \Delta \hat{\mathbf{r}} \equiv \hat{\mathbf{N}} \Delta \hat{\mathbf{r}}=\frac{\ell_{S 0}}{\ell_{S 0}+p_{S 0}} \hat{\mathbf{M}} \Delta \mathbf{r}=\frac{\ell_{S 0}}{\ell_{S 0}+\Delta \ell_{S 0}} \hat{\mathbf{M}} \Delta \hat{\mathbf{r}}_{v}, \\
& \Delta \widehat{\mathbf{r}}_{v} \equiv \Delta \boldsymbol{\rho}_{v} \equiv \mathbf{K}_{m} \Delta \widehat{\mathbf{r}}_{v}=\frac{\ell_{0}+\Delta \ell_{0}}{\ell_{0}} \widehat{\mathbf{M}}_{m} \Delta \widehat{\mathbf{r}}=\frac{\ell_{0}+\Delta \ell_{0}}{\ell_{0}+p_{0}} \widehat{\mathbf{M}}_{m} \Delta \mathbf{r} \\
& =\frac{\ell_{0}+\Delta \ell_{0}}{\ell_{0}+p_{0}+L_{0}} \Delta \rho \\
& \Delta \hat{\mathbf{r}}_{v}=\mathbf{H}_{m} \Delta \hat{\mathbf{r}}_{v}=\frac{\ell_{S 0}+\Delta \ell_{S 0}}{\ell_{S 0}} \hat{\mathbf{M}}_{m} \Delta \hat{\mathbf{r}}=\frac{\ell_{S 0}+\Delta \ell_{S 0}}{\ell_{S 0}+p_{S 0}} \hat{\mathbf{M}}_{m} \Delta \mathbf{r}, \\
& \widehat{\mathbf{M}}_{m} \Delta \mathbf{r}=\frac{\ell_{0}+p_{0}}{\ell_{0}+p_{0}+L_{0}} \Delta \boldsymbol{\rho}=\frac{\ell_{0}+p_{0}}{\ell_{0}+\Delta \ell_{0}} \Delta \boldsymbol{\rho}_{v}, \\
& \Delta \boldsymbol{\rho} \equiv \mathbf{K}_{m} \Delta \rho
\end{aligned}
$$

Assuming $P$ and $P_{0}$ on the object surface, the vector $\Delta \mathbf{r}$ is of particular interest because it contains the information on the surface shape. With the rule $\left(v_{1} \otimes v_{2}\right) v_{3}=v_{1}\left(v_{2} \cdot v_{3}\right)$, where $\mathbf{v}_{1}, \mathbf{v}_{2}$, and $\mathbf{v}_{3}$ are arbitrary vectors of $\mathscr{R}^{3}$, we can decompose $\Delta \mathbf{r}$ into an exterior part along an arbitrary direction c (unit vector) and into an interior part onto a plane normal to $\mathbf{c}$ as follows:

$$
\Delta \mathbf{r}=\mathbf{I} \Delta \mathbf{r}=(\mathbf{C}+\mathbf{c} \otimes \mathbf{c}) \Delta \mathbf{r}=\mathbf{C} \Delta \mathbf{r}+(\mathbf{c} \cdot \Delta \mathbf{r}) \mathbf{c}=\mathbf{C} \Delta \mathbf{r}+\zeta \mathbf{c},
$$

with the normal projector $\mathbf{C}=\mathbf{I}-\mathbf{c} \otimes \mathbf{c}$ and the component $\zeta=\mathbf{c} \cdot \Delta \mathbf{r}$. By choosing $\mathbf{c}=\mathbf{k}_{m}$, we get $\Delta \mathbf{r}=\mathbf{K}_{m} \Delta \mathbf{r}+z \mathbf{k}_{m}$, with the definition $z=\mathbf{k}_{m} \cdot \Delta \mathbf{r}$. We can also decompose $\Delta \mathbf{r}$ into a part along the direction $\mathbf{k}$ and a part onto a plane normal to $\mathbf{k}_{m}$. With Eq. (15), we write

$$
\begin{aligned}
\Delta \mathbf{r} & =\mathbf{I} \Delta \mathbf{r}=\left(\widehat{\mathbf{M}}_{m}+\frac{\mathbf{k} \otimes \mathbf{k}_{m}}{\mathbf{k} \cdot \mathbf{k}_{m}}\right) \Delta \mathbf{r}=\widehat{\mathbf{M}}_{m} \Delta \mathbf{r}+\left(\frac{\mathbf{k}_{m} \cdot \Delta \mathbf{r}}{\mathbf{k} \cdot \mathbf{k}_{m}}\right) \mathbf{k} \\
& =\frac{\ell_{0}+p_{0}}{\ell_{0}+p_{0}+L_{0}} \Delta \boldsymbol{\rho}+\frac{z \mathbf{k}}{\mathbf{k} \cdot \mathbf{k}_{m}} .
\end{aligned}
$$

Introducing Eq. (17) into Eq. (12), the vector $\Delta \hat{\mathbf{r}}$ can also be expressed in function of $\Delta \rho$ and $z$ :

$$
\begin{aligned}
\Delta \hat{\mathbf{r}}= & \frac{\ell_{S 0}\left(\ell_{0}+p_{0}\right)}{\left(\ell_{S 0}+p_{S 0}\right)\left(\ell_{0}+p_{0}+L_{0}\right)} \hat{\mathbf{M}} \Delta \boldsymbol{\rho} \\
& +\frac{\ell_{S 0}}{\ell_{S 0}+p_{S 0}}\left(\frac{\hat{\mathbf{M}} \mathbf{k}}{\mathbf{k} \cdot \mathbf{k}_{m}}\right) z,
\end{aligned}
$$

and the (explicit) general equation for relative moiré [Eq. (9)] becomes (Fig. 7)

$$
\begin{aligned}
\Delta D_{M}= & \frac{1}{\bar{\lambda}} \widehat{\mathbf{g}} \cdot \Delta \widehat{\mathbf{r}}-\frac{1}{\hat{\lambda}} \hat{\mathbf{g}} \cdot \Delta \hat{\mathbf{r}}=\left[\frac{\ell_{0}}{\widehat{\lambda}\left(\ell_{0}+p_{0}\right)} \widehat{\mathbf{g}} \widehat{\mathbf{M}}\right. \\
& \left.-\frac{\ell_{S 0}}{\hat{\lambda}\left(\ell_{S 0}+p_{S 0}\right)} \hat{\mathbf{g}} \hat{\mathbf{M}}\right] \cdot \Delta \mathbf{r}=\mathbf{p}(\mathbf{k}, \mathbf{h}) \cdot \Delta \mathbf{r} \\
= & \frac{\ell_{0}+p_{0}}{\ell_{0}+p_{0}+L_{0}}\left[\frac{\ell_{0}}{\widehat{\lambda}\left(\ell_{0}+p_{0}\right)} \widehat{\mathbf{g}} \cdot \widehat{\mathbf{M}} \Delta \mathbf{\rho}\right. \\
& \left.-\frac{\ell_{S 0}}{\hat{\lambda}\left(\ell_{S 0}+p_{S 0}\right)} \hat{\mathbf{g}} \cdot \hat{\mathbf{M}} \Delta \mathbf{\rho}\right]-\frac{\ell_{S 0}}{\hat{\lambda}\left(\ell_{S 0}+p_{S 0}\right)}\left(\frac{\hat{\mathbf{g}} \cdot \hat{\mathbf{M}} \mathbf{k}}{\mathbf{k} \cdot \mathbf{k}_{m}}\right) z .
\end{aligned}
$$

The meaning of Eq. (19) becomes clear by considering the scalar product of the vector $\Delta \mathbf{r}=\mathbf{r}-\mathbf{r}_{0}$ with the vector $\mathbf{p}=\mathbf{p}(\mathbf{k}, \mathbf{h})$. The displacement vector $\Delta \mathbf{r}$ in space describes the object shape and the sensitivity vector $\mathbf{p}$ depends only on the observing direction $\mathbf{k}$ and on the illuminating direction $\mathbf{h}$ for a given optical setup. Note that this definition is very much an analog to the definition of the fringe order in holographic and speckle interferometry. ${ }^{14,17}$ The moiré surfaces in space are then described by a set of vectors $\Delta \mathbf{r}=\Delta \mathbf{r}_{M}=\mathbf{r}_{M}-\mathbf{r}_{0}$ for which $\Delta D_{M}=\not$. Because the sensitivity vector $\mathbf{p}(\mathbf{k}, \mathbf{h})$ is not constant, we must pay particular attention to the fact that the moiré surfaces in space may generally be curved.

\subsection{Special Case of Shadow Moiré with Collimated Light}

This case concerns the projection and observation with collimated rays (parallel rays) through the same grating on the same grid plane, where the optical axes of the camera and of the projector are not parallel. This particular case can be obtained from the general case [Eq. (19)] by taking a limit when $\ell_{0} \rightarrow \infty$ and $\ell_{S 0} \rightarrow \infty$ while keeping $\overline{\mathbf{g}} / \widehat{\lambda} \equiv \hat{\mathrm{g}} / \hat{\lambda}=\phi$. In our model (Fig. 8), this means that $R$ and $S$ go to infinity along the directions $\mathbf{k}_{m}$ and $-\mathbf{h}_{m}$, respectively, while the 


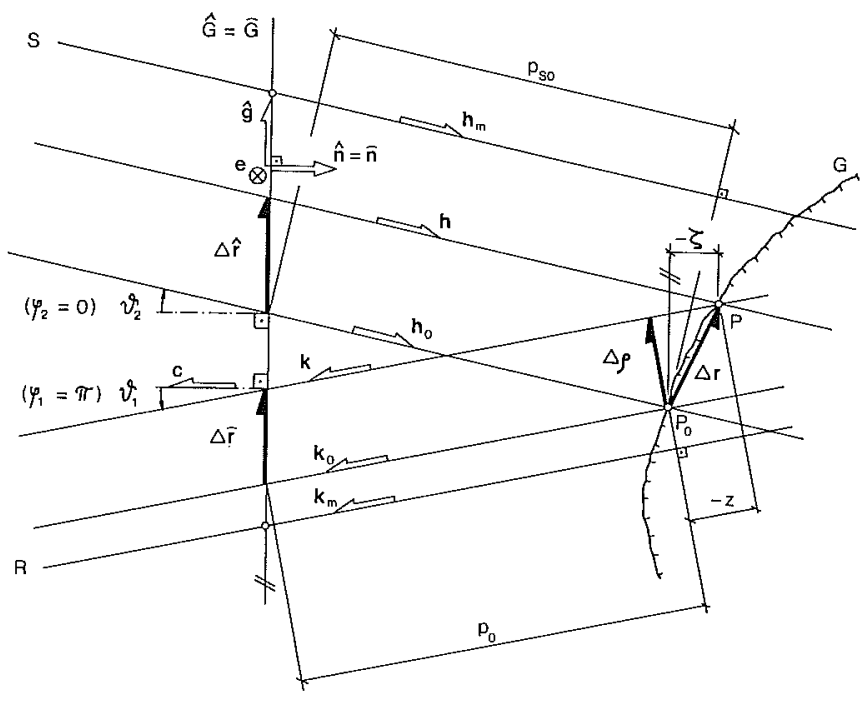

Fig. 8 Special case of shadow moiré with collimated light.

position of the grid-plane $\widehat{G}=\hat{G}$ remains unchanged relatively to the object surface $G$. Focusing near the object and by knowing that $p_{0}, p_{S 0}, L_{0}$, and $L_{S 0}$ remain finite, we have with $\mathbf{k}_{m} \neq-\mathbf{h}_{m}$ and by choosing $\mathbf{c}=-\widehat{\mathbf{n}}=-\hat{\mathbf{n}}$,

$\lim _{\ell_{0} \rightarrow \infty} \frac{\ell_{0}+p_{0}}{\ell_{0}+p_{0}+L_{0}}=\lim _{\ell_{0} \rightarrow \infty} \frac{\ell_{0}}{\ell_{0}+p_{0}}=\lim _{\ell_{S 0} \rightarrow \infty} \frac{\ell_{S 0}}{\ell_{S 0}+p_{S 0}}=1$,

$$
\mathbf{k}=\mathbf{k}_{m}, \quad \mathbf{h}=\mathbf{h}_{m},
$$

$\mathbf{C}=\widehat{\mathbf{N}}=\hat{\mathbf{N}}, \quad \mathbf{K}_{m} \neq \mathbf{H}_{m}, \widehat{\mathbf{M}}=\mathbf{I}-\frac{\mathbf{k}_{m} \otimes \mathbf{c}}{\mathbf{k}_{m} \cdot \mathbf{c}}, \hat{\mathbf{M}}=\mathbf{I}-\frac{\mathbf{h}_{m} \otimes \mathbf{c}}{\mathbf{h}_{m} \cdot \mathbf{c}}$

With $(\widehat{\mathbf{M}}-\hat{\mathbf{M}}) \mathbf{C}=0, \widehat{\mathbf{M k}}_{m}=0$, and $\Delta \mathbf{r}=\mathbf{C} \Delta \mathbf{r}+\zeta \mathbf{c}=\Delta \boldsymbol{\rho}+$ $z \mathbf{k}_{m}$, Eq. (19) becomes

$\Delta D_{M}=\frac{\zeta}{\hat{\lambda}} \hat{\mathbf{g}} \cdot(\widehat{\mathbf{M}}-\hat{\mathbf{M}}) \mathbf{c}=\frac{1}{\hat{\lambda}} \hat{\mathbf{g}} \cdot\left[(\widehat{\mathbf{M}}-\hat{\mathbf{M}}) \Delta \mathbf{\rho}-z \hat{\mathbf{M}} \mathbf{k}_{m}\right]$.

In this particular case, the moiré surfaces $\Delta D_{M}=\varnothing$ in space are given by a set of "equally spaced" parallel planes normal to the direction of the constant sensitivity vector $\mathbf{p}=$ $\hat{\mathbf{g}}(\overline{\mathbf{M}}-\hat{\mathbf{M}}) / \hat{\lambda}$.

We now define an orthogonal basis $(\hat{\mathbf{g}}, \mathbf{e}, \mathbf{c})$ where e $\equiv \mathbf{C e}=\mathbf{c} \times \hat{\mathrm{g}}$ is a unit vector in the grid plane and write the two vectors $\mathbf{k}_{m}$ and $\mathbf{h}_{m}$ in components:

$$
\begin{aligned}
\mathbf{k}_{m} & =\left(\mathbf{k}_{m} \cdot \hat{\mathbf{g}}\right) \hat{\mathbf{g}}+\left(\mathbf{k}_{m} \cdot \mathbf{e}\right) \mathbf{e}+\left(\mathbf{k}_{m} \cdot \mathbf{c}\right) \mathbf{c} \\
& =\hat{\mathbf{g}} \sin \vartheta_{1} \cos \varphi_{1}+\mathbf{e} \sin \vartheta_{1} \sin \varphi_{1}+\mathbf{c} \cos \vartheta_{1}, \\
\mathbf{h}_{m} & =\left(\mathbf{h}_{m} \cdot \hat{\mathbf{g}}\right) \hat{\mathbf{g}}+\left(\mathbf{h}_{m} \cdot \mathbf{e}\right) \mathbf{e}+\left(\mathbf{h}_{m} \cdot \mathbf{c}\right) \mathbf{c} \\
& =-\hat{\mathbf{g}} \sin \vartheta_{2} \cos \varphi_{2}-\mathbf{e} \sin \vartheta_{2} \sin \varphi_{2}-\mathbf{c} \cos \vartheta_{2} .
\end{aligned}
$$

Introducing Eqs. (21) and (23) into Eq. (22), we get the following expression [Eq. (24)] for shadow moiré. By considering the particular situation of Fig. 8, where $\varphi_{1}=\pi$ and $\varphi_{2}=0$, we become the well-known following second expression [Eq. (25)] [Eq. (1) in Ref. 1, Eq. (6) in Ref. 3, Eq. (27) in Ref. 4, and Eq. (5) in Ref. 5]. Both Eqs. (24) and (25) describe the level lines of the object shape relatively to a plane normal to $\mathrm{c}$ passing through the point $P_{0}$ :

$\zeta=\frac{-\hat{\lambda} \Delta D_{M}}{\tan \vartheta_{1} \cos \varphi_{1}-\tan \vartheta_{2} \cos \varphi_{2}}$

$$
\zeta=\frac{\hat{\lambda} \Delta D_{M}}{\tan \vartheta_{1}+\tan \vartheta_{2}} .
$$

\subsection{Special Case of Projection Moiré with Collimated Light}

This case concerns projection and observation with collimated rays, where the two grid planes are perpendicular to their respective optical axes, which are generally not parallel. This particular case can also be obtained from the general case [Eq. (19)] by taking a limit when $\ell_{0} \rightarrow \infty$ and $\ell_{S 0} \rightarrow \infty$ while keeping $\hat{\mathbf{g}} / \widehat{\lambda}=\phi$ and $\hat{\mathbf{g}} / \hat{\lambda}=\phi$. In our model (Fig. 9), this means that $R$ and $S$ go to infinity along the directions $\mathbf{k}_{m}$ and $-\mathbf{h}_{m}$, respectively, while the position of the grid planes $\widehat{G}$ and $\hat{G}$ remain unchanged relative to the object surface $G$. Focusing near the object and knowing that $p_{0}, p_{S 0}, L_{0}$, and $L_{S 0}$ remain finite, Eqs. (20) are still valid in this case. With $\widehat{\mathbf{n}}=-\mathbf{k}_{m}, \hat{\mathbf{n}}=\mathbf{h}_{m}, \widehat{\mathbf{N}}=\mathbf{K}_{m}, \hat{\mathbf{N}}=\mathbf{H}_{m}, \widehat{\mathbf{M}}=\mathbf{K}_{m}, \hat{\mathbf{M}}=\mathbf{H}_{m}$, and $\Delta \mathbf{r}=\Delta \boldsymbol{\rho}+z \mathbf{k}_{m}$, Eq. (19) becomes $^{18}$

$$
\begin{aligned}
\Delta D_{M}= & \left(\frac{1}{\bar{\lambda}} \widehat{\mathbf{g}} \mathbf{K}_{m}-\frac{1}{\hat{\lambda}} \hat{\mathbf{g}} \mathbf{H}_{m}\right) \cdot \Delta \mathbf{r}=\frac{\mathbf{1}}{\bar{\lambda}} \widehat{\mathbf{g}} \cdot \mathbf{K}_{m} \Delta \boldsymbol{\rho} \\
& -\frac{1}{\hat{\lambda}} \hat{\mathbf{g}} \cdot \mathbf{H}_{m} \Delta \mathbf{\rho}-\frac{1}{\hat{\lambda}}\left(\hat{\mathbf{g}} \cdot \mathbf{H}_{m} \mathbf{k}_{m}\right) z .
\end{aligned}
$$

In this particular case, the moire surfaces $\Delta D_{M}=\varnothing$ in space are given by a set of "equally spaced" parallel planes normal to the direction of the constant sensitivity vector $\mathbf{p}=$ $\left(\widehat{\mathbf{g}} \mathbf{K}_{m} / \widehat{\lambda}\right)-\left(\hat{\mathbf{g}} \mathbf{H}_{m} / \hat{\lambda}\right)$

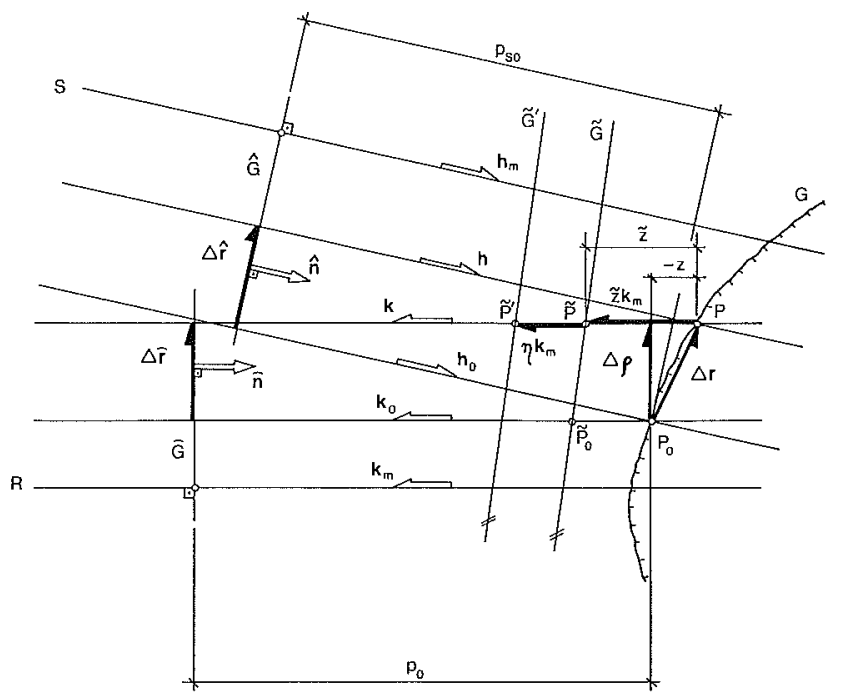

Fig. 9 Special case of projection moiré with collimated light. 
To determine the geometrical terms in Eq. (26), we introduce a calibration plane $\tilde{G}$ in the vicinity of the object ${ }^{6,7}$ and write the corresponding expression for relative moiré in point $\tilde{P}$ relative to point $P_{0}$ :

$$
\begin{aligned}
\Delta \tilde{D}_{M}= & \tilde{D}_{M}-D_{M 0}=\left(\frac{1}{\widehat{\lambda}} \mathbf{g} \mathbf{K}_{m}-\frac{1}{\hat{\lambda}} \hat{\mathbf{g}} \mathbf{H}_{m}\right) \\
& \cdot\left(\Delta \mathbf{r}+\tilde{z} \mathbf{k}_{m}\right)+\left(\tilde{\Psi}-\Psi_{0}\right),
\end{aligned}
$$

where $\tilde{z} \mathbf{k}_{m}$ is the vector in the observing direction from point $P$ on the object surface to point $\tilde{P}$ on the calibration plane. Assuming that $\tilde{\Psi}=\Psi_{0}$ and knowing that $\mathbf{K}_{m} \mathbf{k}_{m}=0$, by taking the difference between Eqs. (26) and (27), we have

$\delta \tilde{D}_{M}=\Delta \tilde{D}_{M^{--}} \Delta D_{M}=-\frac{\tilde{z}}{\hat{\lambda}} \hat{\mathbf{g}} \cdot \mathbf{H}_{m} \mathbf{k}_{m}=-A_{0} \tilde{z}$,

with $\delta \tilde{D}_{M}$ describing the difference of the moire fringe order between points $\tilde{P}$ and $P$. The difference of the fringe order will now be simply called difference moiré. As we can see, Eq. (28) for $\delta \tilde{D}_{M}$ gives the level lines of the object shape respectively to the calibration plane $\tilde{G}$ and the observing direction $\mathbf{k}_{m}$. To get the still unknown proportionality factor $A_{0}=\hat{\mathbf{g}} \cdot \mathbf{H}_{m} \mathbf{k}_{m} / \hat{\lambda}=\phi$, we can translate the calibration plane along the observing direction by a known amount $\eta$ and write with $\tilde{\Psi}^{\prime}=\Psi_{0}$ for the new position $\tilde{G}^{\prime}$

$$
\delta \tilde{D}_{M}^{\prime}=\Delta \tilde{D}_{M}^{\prime}-\Delta D_{M}=-\frac{(\tilde{z}+\eta)}{\hat{\lambda}} \hat{\mathbf{g}} \cdot \mathbf{H}_{m} \mathbf{k}_{m}=-A_{0}(\tilde{z}+\eta),
$$

where $\delta \tilde{D}_{M}^{\prime}$ describes the difference moiré between points $\tilde{P}^{\prime}$ and $P$ and where $\Delta \tilde{D}_{M}^{\prime}=\tilde{D}_{M}^{\prime}-D_{M 0}$ is the relative moire in point $\tilde{P}^{\prime}$ relative to point $P_{0}$. Note that the vector $\eta \mathbf{k}_{m}$ is given and describes the translation of the calibration plane. By solving the system of Eqs. (28) and (29), we get

$$
\begin{gathered}
\tilde{z}=\frac{\eta \delta \tilde{D}_{M}}{\delta \tilde{D}_{M}^{\prime}-\delta \tilde{D}_{M}}=\frac{\eta\left(\tilde{D}_{M}-D_{M}\right)}{\tilde{D}_{M}^{\prime}-\tilde{D}_{M}}, \\
A_{0}=\frac{\delta \tilde{D}_{M}-\delta \tilde{D}_{M}^{\prime}}{\eta}=\frac{\tilde{D}_{M}-\tilde{D}_{M}^{\prime}}{\eta} .
\end{gathered}
$$

Let us now consider the more special planar case of Fig. 10, where $\quad \widehat{\lambda}=\hat{\lambda}, \quad \overline{\mathbf{g}} \perp\left(\mathbf{k}_{m} \times \mathbf{h}_{m}\right), \quad \hat{\mathbf{g}} \perp\left(\mathbf{k}_{m} \times \mathbf{h}_{m}\right), \quad \overline{\mathbf{g}} \cdot \hat{\mathbf{g}}>0$ and $\mathbf{k}_{m} \cdot \mathbf{h}_{m}<0$. By writing $\mathbf{c}=(\widehat{\mathbf{g}}-\hat{\mathbf{g}}) /|\widehat{\mathbf{g}}-\hat{\mathbf{g}}|=(\widehat{\mathbf{g}}-\hat{\mathbf{g}}) / 2$ $\sin \vartheta$ with $\widehat{\mathbf{g}} \cdot \hat{\mathbf{g}}=-\mathbf{k}_{m} \cdot \mathbf{h}_{m}=\cos 2 \vartheta$ and knowing that $\widehat{\mathbf{g}} \equiv$ $\mathbf{K}_{m} \mathbf{g}$ and $\hat{\mathbf{g}}=\mathbf{H}_{m} \hat{\mathbf{g}}$, with Eqs. (16) and (26) we get the following well-known expression for $\Delta D_{M}$ [Eq. (46) of Ref. 1 and Eq. (8) of Ref. 5] describing the level lines respective to a plane normal to $\mathrm{c}$ passing through the point $P_{0}$

$$
\begin{aligned}
\Delta D_{M} & =\frac{1}{\hat{\lambda}}\left(\widehat{\mathbf{g}} \mathbf{K}_{m}-\hat{\mathbf{g}} \mathbf{H}_{m}\right) \cdot(\mathbf{C} \Delta \mathbf{r}+\zeta \mathbf{c})=\frac{2 \zeta \sin \vartheta}{\hat{\lambda}} \\
& \rightarrow \zeta=\frac{\hat{\lambda} \Delta D_{M}}{2 \sin \vartheta} .
\end{aligned}
$$

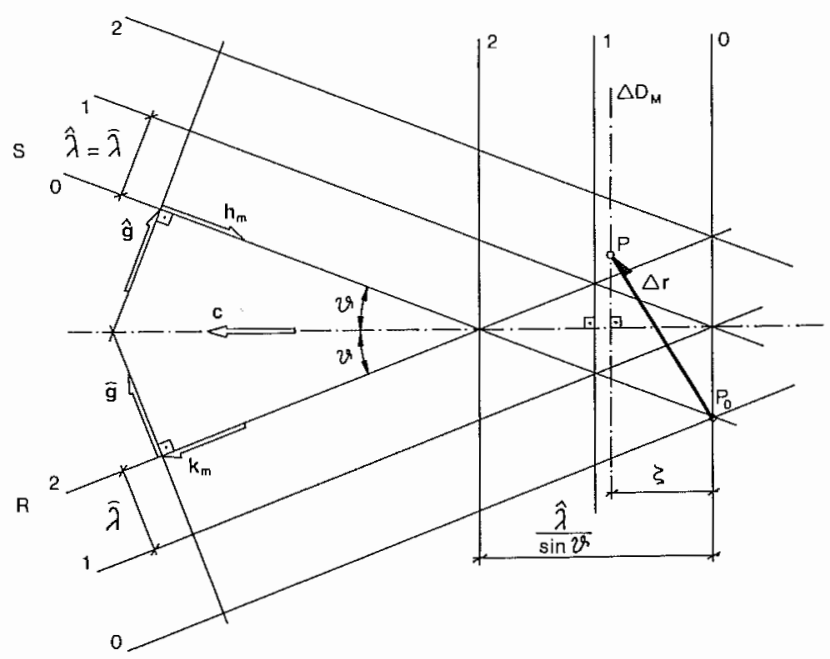

Fig. 10 Special planar case of projection moiré with collimated light.

In the nonplanar case where $\hat{\mathbf{g}} \cdot \hat{\mathbf{g}} \neq-\mathbf{k}_{m} \cdot \mathbf{h}_{m}, \mathrm{Eq}$. (31) is still valid if $2 \vartheta$ describes the angle between the two vectors $\widehat{\mathbf{g}}$ and $\hat{\mathbf{g}}$.

\section{General Expression of Difference Moiré}

\subsection{Theoretical Calibration of a Moiré Setup}

To illustrate the calibration procedure in a projection moiré experiment, we consider the general setup of Fig. 11, where the two grid planes are perpendicular to their respective optical axes, which means $\overline{\mathbf{n}}=-\mathbf{k}_{m}, \hat{\mathbf{n}}=\mathbf{h}_{m}$, and where $\Delta \ell_{0}=\Delta \ell_{S 0}=0$. In place of the object, we consider a calibration plane $G$, which can be moved normally to its surface. In a real experiment, neither the position of the centers of projection $(R$ and $S$ ) nor the distance to the object surface are known exactly. We can therefore choose in the 3 -D space some reference point $P_{0}$ that will play an important role in the calibration process. All the geometrical quantities are expressed relative to this point and, after being inserted in the theoretical equations, are useful to estimate the calibration constants and the correction terms. Translating the calibration plane by a known amount along its unit normal $\tilde{\mathbf{n}}$, the fringe order in each point as observed by the camera changes. With Eq. (19), which means with $\Delta D_{M}=\mathbf{p}(\mathbf{k}, \mathbf{h}) \cdot \Delta \mathbf{r}$, we can write by definition the general expression for the relative moiré in point $\tilde{P}$ relative to the same point $P_{0}$

$$
\begin{aligned}
\Delta \tilde{D}_{M}= & \Delta D_{M}+\delta \tilde{D}_{M}=\left[\frac{\ell_{0}}{\hat{\lambda}\left(\ell_{0}+p_{0}\right)} \widehat{\mathbf{g}} \hat{\tilde{\mathbf{M}}}-\frac{\ell_{S 0}}{\hat{\lambda}\left(\ell_{S 0}+p_{S 0}\right)} \hat{\mathbf{g}} \hat{\tilde{\mathbf{M}}}\right] \\
& \cdot(\Delta \mathbf{r}+\eta \mathbf{k}),
\end{aligned}
$$

with the two oblique projectors $\widetilde{\mathbf{M}}=\mathbf{I}-[\tilde{\mathbf{k}} \otimes) \tilde{\mathbf{n}} /(\tilde{\mathbf{k}} \cdot \tilde{\mathbf{n}})]$ and $\hat{\mathbf{M}}=\mathbf{I}-[\tilde{\mathbf{h}} \otimes \hat{\mathbf{n}} /(\tilde{\mathbf{h}} \cdot \hat{\mathbf{n}})]$ and where $\delta \tilde{D}_{M}=\Delta \tilde{D}_{M}-\Delta D_{M}$ is the difference moiré giving the difference in fringe order between the two states $\widetilde{G}$ and $G$ of the calibration plane as viewed by a pixel of the camera.

To discuss the effects that lead to the behavior of the difference moiré across the field of view, we first have written Eq. (32) in general terms of Fig. 7. Because the observing 


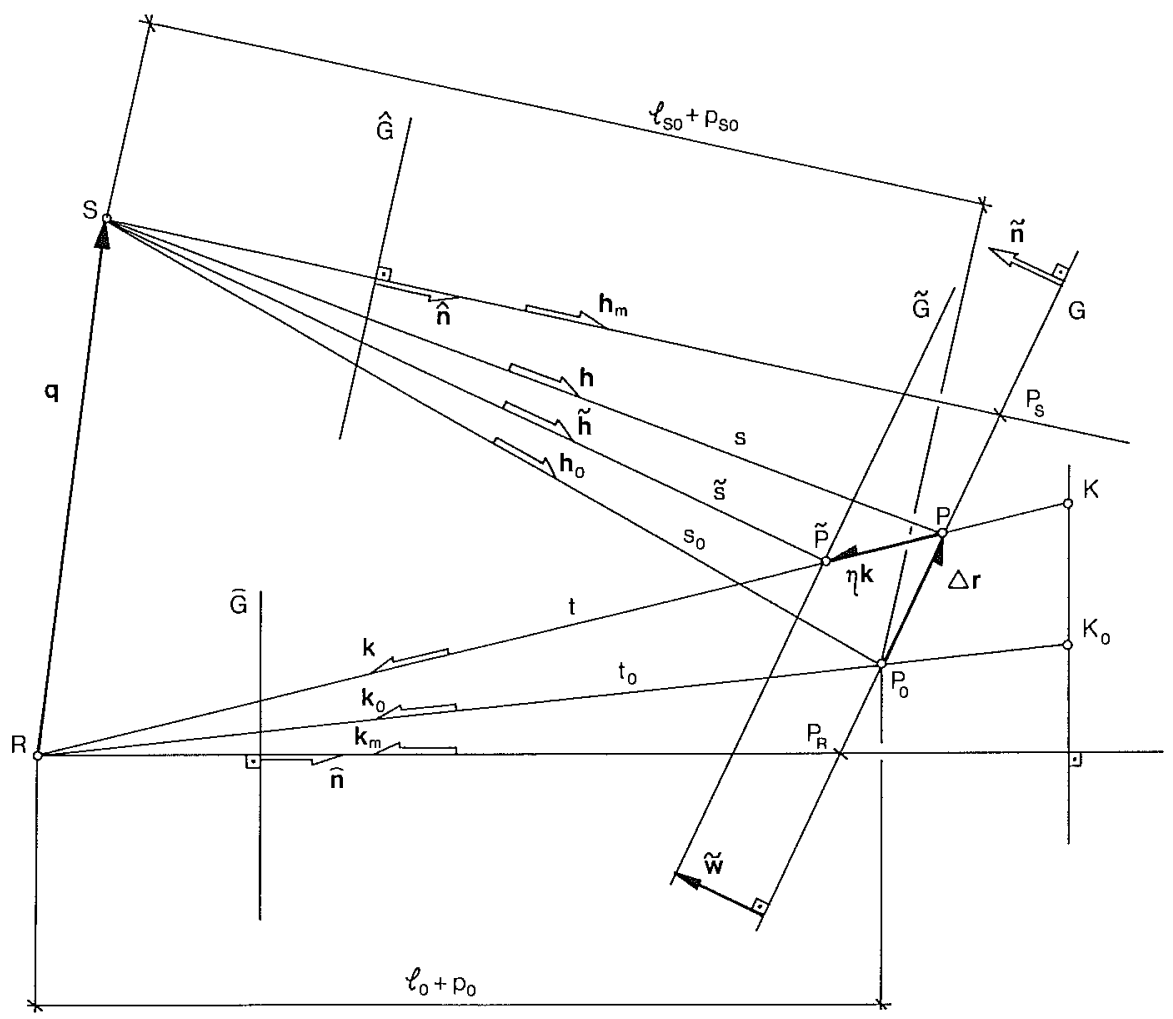

Fig. 11 General moiré setup for the calibration process.

direction is the same for each pixel, we have $\tilde{\mathbf{k}}=\mathbf{k}$ and $\widetilde{\mathbf{M}}=\overrightarrow{\mathbf{M}}$. Therefore the difference moiré is only given by the projector grating (the camera grating is however still needed because of our pixel resolution) and we have with $\widehat{\mathbf{M}} \mathbf{k}=0$ and the definition $\lambda=\hat{\lambda}\left(\ell_{S 0}+p_{S 0}\right) / \ell_{S 0}$ :

$$
\delta \tilde{D}_{M}=\frac{1}{\lambda}(\hat{\mathbf{g}} \hat{\mathbf{M}}-\hat{\mathbf{g}} \hat{\mathbf{M}}) \cdot \Delta \mathbf{r}-\frac{\eta}{\lambda} \hat{\mathbf{g}} \cdot \hat{\tilde{\mathbf{M}}} \mathbf{k}
$$

Note that in the particular case of parallel projection $\tilde{\mathbf{h}}=\mathbf{h}$, we have $\hat{\mathbf{M}}=\hat{\mathbf{M}}$ and only the last term gives a contribution. Returning to our general case, Eq. (33), which does not depend on $\overline{\mathbf{g}} / \widehat{\lambda}$, can be written in a slightly different form by using the vector definitions $\mathbf{S} \tilde{\mathbf{P}}=\tilde{s} \tilde{\mathbf{h}}=s \mathbf{h}+\eta \mathbf{k}$ and $\mathbf{S P}=s \mathbf{h}=s_{0} \mathbf{h}_{0}+\Delta \mathbf{r}$. The general expression for difference moiré (Fig. 11) is

$\delta \tilde{D}_{M}=-\frac{\eta}{\lambda}(\hat{\mathbf{g}} \cdot \hat{\mathbf{M}} \mathbf{k}) \frac{s_{0} \mathbf{h}_{0} \cdot \hat{\mathbf{n}}}{\left(s_{0} \mathbf{h}_{0}+\Delta \mathbf{r}+\eta \mathbf{k}\right) \cdot \hat{\mathbf{n}}}$,

with

$\hat{\mathbf{M}}=\mathbf{I}-\frac{\left(s_{0} \mathbf{h}_{0}+\Delta \mathbf{r}\right) \otimes \hat{\mathbf{n}}}{\left(s_{0} \mathbf{h}_{0}+\Delta \mathbf{r}\right) \cdot \hat{\mathbf{n}}}$,

where $s_{0} \mathbf{h}_{0} \cdot \hat{\mathbf{n}}=\ell_{s 0}+p_{s 0}$. The incremental vector $\Delta \mathbf{r}$ and the first multiplicative part $\hat{\mathbf{g}} \cdot \hat{\mathbf{M}} \mathbf{k} / \lambda$ in Eq. (34) are only pixel dependent, which means they are constant for a fixed observing direction $\mathbf{k}$. The second multiplicative part $s_{0} \mathbf{h}_{0} \cdot \hat{\mathbf{n}} /$ $\left[\left(s_{0} \mathbf{h}_{0}+\Delta \mathbf{r}+\eta \mathbf{k}\right) \cdot \hat{\mathbf{n}}\right]$ depends on both the observing direction and the translation of the calibration plane. With the small varying vector $\eta \mathbf{k}$ and the unit normal $\tilde{\mathbf{n}}$ to the calibration plane, we introduce the incremental value $\tilde{z}=\eta \mathbf{k} \cdot \tilde{\mathbf{n}}$, which is constant over the whole calibration plane. In our experimental setup, the translation vector $\tilde{\mathbf{w}}=\tilde{z} \tilde{\mathbf{n}}$ describes the global translation of the calibration plane. With the vector definitions $\mathbf{P}_{0} \mathbf{R}=t_{0} \mathbf{k}_{0}$ and $\mathbf{P R}=t \mathbf{k}=t_{0} \mathbf{k}_{0}-\Delta \mathbf{r}$, we write

$\eta \mathbf{k}=\left(\frac{\eta \mathbf{k} \cdot \tilde{\mathbf{n}}}{t \mathbf{k} \cdot \tilde{\mathbf{n}}}\right) t \mathbf{k}=\frac{\tilde{z}\left(t_{0} \mathbf{k}_{0}-\Delta \mathbf{r}\right)}{\left(t_{0} \mathbf{k}_{0}-\Delta \mathbf{r}\right) \cdot \tilde{\mathbf{n}}}=\frac{\tilde{z}}{\tilde{c}}\left(t_{0} \mathbf{k}_{0}-\Delta \mathbf{r}\right)$

where $\tilde{c}=\left(t_{0} \mathbf{k}_{0}-\Delta \mathbf{r}\right) \cdot \tilde{\mathbf{n}}$. Because the calibration plane has no curvature and by taking into account that $P_{0}$ is assumed to lie in the calibration plane in its initial position, we have $\Delta \mathbf{r} \cdot \tilde{\mathbf{n}}=0$. It follows that the value of $\tilde{c}=c_{0}=t_{0} \mathbf{k}_{0} \cdot \tilde{\mathbf{n}}$ is constant in this case. We can now define three new variables $x_{1}, x_{2}$, and $x_{3}$, fully describing the change of $\delta \tilde{D}_{M}$ :

$x_{1}=\frac{\tilde{z}}{\tilde{c}}=\frac{\tilde{z}}{c_{0}}=\frac{\tilde{z}}{t_{0} \mathbf{k}_{0} \cdot \tilde{\mathbf{n}}}, \quad x_{2}=\frac{\Delta \mathbf{r} \cdot \hat{\mathbf{n}}}{s_{0} \mathbf{h}_{0} \cdot \hat{\mathbf{n}}}, \quad x_{3}=\frac{\Delta \mathbf{r} \cdot \hat{\mathbf{g}}}{s_{0} \mathbf{h}_{0} \cdot \hat{\mathbf{n}}}$,

and write, with the constant parameter $a=t_{0} \mathbf{k}_{0} \cdot \hat{\mathbf{n}} /\left(s_{0} \mathbf{h}_{0} \cdot \hat{\mathbf{n}}\right)$ and the constant vector $\mathbf{q}=-s_{0} \mathbf{h}_{0}-t_{0} \mathbf{k}_{0}$, the following exact relations:

$\frac{\eta \mathbf{k} \cdot \hat{\mathbf{n}}}{s_{0} \mathbf{h}_{0} \cdot \hat{\mathbf{n}}}=\frac{\tilde{z}}{\tilde{c}}\left(\frac{t_{0} \mathbf{k}_{0} \cdot \hat{\mathbf{n}}-\Delta \mathbf{r} \cdot \hat{\mathbf{n}}}{s_{0} \mathbf{h}_{0} \cdot \hat{\mathbf{n}}}\right)=x_{1}\left(a-x_{2}\right)$,

$\frac{s_{0} \mathbf{h}_{0} \cdot \hat{\mathbf{n}}}{\left(s_{0} \mathbf{h}_{0}+\Delta \mathbf{r}+\eta \mathbf{k}\right) \cdot \hat{\mathbf{n}}}=\frac{1}{1+(\Delta \mathbf{r} \cdot \hat{\mathbf{n}}) /\left(s_{0} \mathbf{h}_{0} \cdot \hat{\mathbf{n}}\right)+(\eta \mathbf{k} \cdot \hat{\mathbf{n}}) /\left(s_{0} \mathbf{h}_{0} \cdot \hat{\mathbf{n}}\right)}$ 


$$
\begin{gathered}
=\frac{1}{1+a x_{1}+x_{2}-x_{1} x_{2}}, \\
-\frac{\eta}{\lambda} \hat{\mathbf{g}} \cdot \hat{\mathbf{M}} \mathbf{k}=-\frac{\hat{\mathbf{g}}}{\lambda} \cdot\left[\mathbf{I}-\frac{\left(s_{0} \mathbf{h}_{0}+\Delta \mathbf{r}\right) \otimes \hat{\mathbf{n}}}{\left(s_{0} \mathbf{h}_{0}+\Delta \mathbf{r}\right) \cdot \hat{\mathbf{n}}}\right] \tilde{z}\left(t_{0} \mathbf{k}_{0}-\Delta \mathbf{r}\right) \\
=\frac{x_{1}}{\lambda}\left[\mathbf{q} \cdot \hat{\mathbf{g}}+\left(\frac{1+a}{1+x_{2}}\right) s_{0} \mathbf{h}_{0} \cdot\left(\hat{\mathbf{g}}+x_{3} \hat{\mathbf{n}}\right)\right],
\end{gathered}
$$

to get finally the exact expression for $\delta \tilde{D}_{M}=\delta \tilde{D}_{M}\left(x_{1}, x_{2}, x_{3}\right)$ :

$$
\begin{aligned}
\delta \tilde{D}_{M}= & \frac{x_{1}}{\lambda\left(1+a x_{1}+x_{2}-x_{1} x_{2}\right)} \\
& \times\left[\mathbf{q} \cdot \hat{\mathbf{g}}+\left(\frac{1+a}{1+x_{2}}\right) s_{0} \mathbf{h}_{0} \cdot\left(\hat{\mathbf{g}}+x_{3} \hat{\mathbf{n}}\right)\right] .
\end{aligned}
$$

\subsection{Experimental and Numerical Verification}

To show how the different terms in Eq. (38) contribute to the calibration variation, we analytically simulate a calibration process and compare the results with an analog experiment. For that purpose, we introduce a Cartesian system $\left(P_{0}, \tilde{x}, \tilde{y}, \tilde{z}\right)$, where the $\tilde{x}$ and $\tilde{y}$ axes lie on the calibration plane in its initial position and where the $\tilde{z}$ axis is parallel to $\tilde{\mathbf{n}}$. Parallel to the $\tilde{x}$ and $\tilde{y}$ axes, we respectively introduce the unit vectors $\mathbf{e}_{1}$ and $\mathbf{e}_{2}$. For better visuality, we assume that the four vectors $\hat{\mathbf{g}}, \hat{\mathbf{n}}, \tilde{\mathbf{n}}$, and $\hat{\mathbf{n}}$ are coplanar and lie in the horizontal plane, the calibration plane being vertical and the $\tilde{x}$ axis being horizontal (Fig. 12). Without restricting the generality, we also assume that the two optical axes (camera and projector) intersect the calibration plane in the same point $P_{0}$, which means $\mathbf{h}_{0}=\mathbf{h}_{m}=\hat{\mathbf{n}}$ and $s_{0} \mathbf{h}_{0} \cdot \hat{\mathbf{g}}=0$. To explain the meaning of Eq.(38), we write a linear approximation considering that $\left|x_{1}\right| \ll<1$ and $\left|x_{2}\right| \ll<1$, and with $(1+a)\left(s_{0} \mathbf{h}_{0}\right.$. $\hat{\mathbf{n}})=-\mathbf{q} \cdot \hat{\mathbf{n}}$, it becomes $\delta \tilde{D}_{M} \simeq \delta \tilde{D}_{M L}=\frac{x_{1}}{\lambda}\left(\mathbf{q} \cdot \hat{\mathbf{g}}-x_{3} \mathbf{q} \cdot \hat{\mathbf{n}}\right)$

As already mentioned, the exact expression of Eq. (38) for the difference moiré $\delta \tilde{D}_{M}$ describes the difference of the fringe order in some point $K$ for a known variation in depth of field $\tilde{z}$. Note that $K$ is a point in the object plane of the optical system of the camera and is associated to the corresponding pixel in the image plane. The first term $x_{1} \mathbf{q} \cdot \hat{\mathbf{g}} / \lambda$ of Eq. (39) gives a linear approximation of the difference moiré in point $K_{0}$ for a small change in depth of field and represents the constant contribution for the whole calibration plane. The second term $-x_{1} x_{3} \mathbf{q} \cdot \hat{\mathbf{n}} / \lambda$ gives, for a fixed variation in depth of field, a linear approximation of the variation of the difference moiré across the field of view when we start from the point $K_{0}$ and go to some other point $K$. In our experimental setup, we have approximately adjusted $P_{0}$ on the observing direction $\mathbf{k}_{m}$, which means that $\mathbf{k}_{0} \simeq \mathbf{k}_{m}=-\overline{\mathbf{n}}$. We used a white-painted mirror as calibration plane $G$, which was installed on a translation stage to move it normally to its surface. It was illuminated by a projector with a grating on a glass slide and observed through a CCD-interline transfer camera, which pixel columns served as reference grating. The experimental numerical values corresponding to the optical setup of Fig. 12 needed to compute Eqs. (38) and (39) are given as follows:

\begin{tabular}{lll} 
& \multicolumn{1}{c}{ Projector } & \multicolumn{1}{c}{ Camera } \\
\hline $\begin{array}{l}\text { Distance } P_{0} \text { to } \\
\text { projection center }\end{array}$ & $\ell_{S O}+p_{S O} \simeq 304.0 \mathrm{~mm}$ & $\ell_{0}+p_{0} \simeq 359.0 \mathrm{~mm}$ \\
$\begin{array}{l}\text { Distance grating to } \\
\text { projection center }\end{array}$ & $\ell_{S O} \simeq 73.97 \mathrm{~mm}$ & $\ell_{0}=29.92 \mathrm{~mm}$ \\
$\begin{array}{l}\text { Inclination of } \\
\text { optical axis }\end{array}$ & $\alpha_{S} \simeq 5.15 \mathrm{deg}$ & $\alpha_{R} \simeq 6.70 \mathrm{deg}$ \\
Pitch of grating & $\hat{\lambda}=50 \mu \mathrm{m}=0.050 \mathrm{~mm}$ & $\hat{\lambda}=17 \mu \mathrm{m}=0.017 \mathrm{~mm}$ \\
\hline
\end{tabular}

*Respectively calculated with the magnification factors 4.11 and 12.0 on the optical axes.

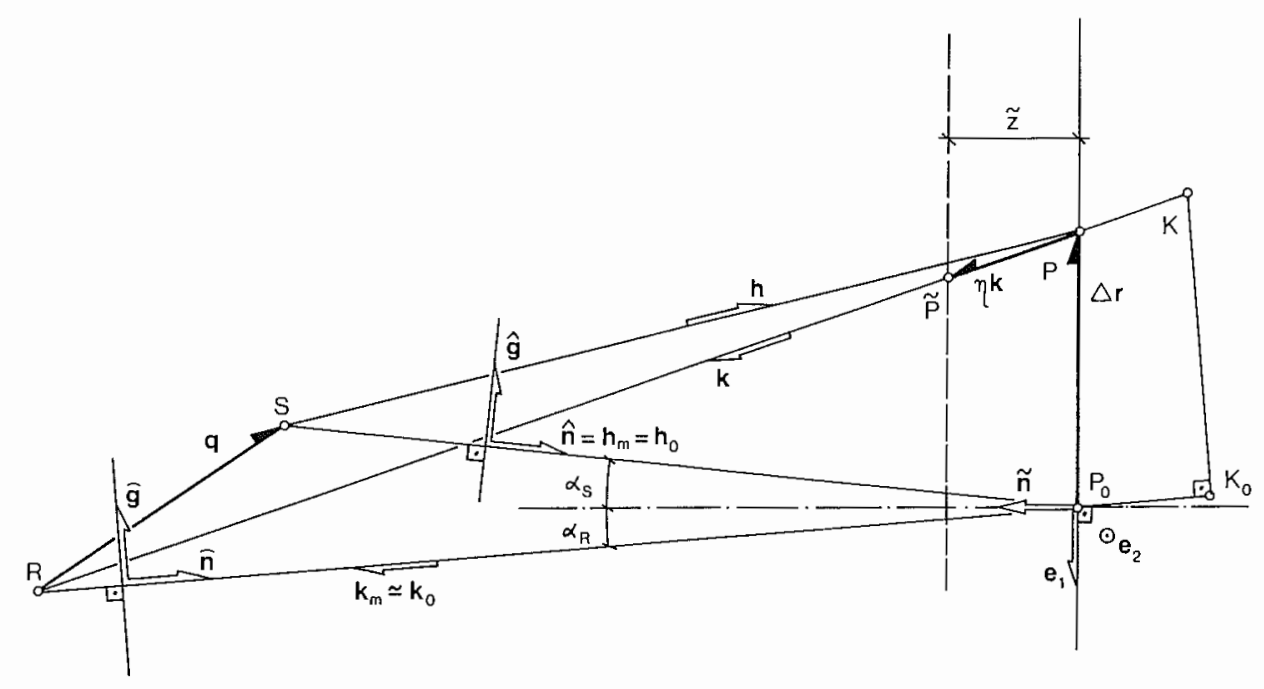

Fig. 12 Setup for the numerical verification. 
$-t_{0} \mathbf{k}_{0} \cdot \overline{\mathbf{n}}=\ell_{0}+p_{0}=359.0 \mathrm{~mm}$

$s_{0} \mathbf{h}_{0} \cdot \hat{\mathbf{n}}=\ell_{S 0}+p_{S 0}=304.0 \mathrm{~mm}$,

$t_{0} \mathbf{k}_{0} \cdot \hat{\mathbf{n}}=-\left(\ell_{0}+p_{0}\right) \cos \left(\alpha_{R}+\alpha_{S}\right)$

$=-359.0 \cos 11.85=-351.3 \mathrm{~mm}$,

$c_{0}=t_{0} \mathbf{k}_{0} \cdot \tilde{\mathbf{n}}=\left(\ell_{0}+p_{0}\right) \cos \alpha_{R}$

$=359.0 \cos 6.70=356.5 \mathrm{~mm}$

$\mathbf{q} \cdot \hat{\mathbf{g}}=-s_{0} \mathbf{h}_{0} \cdot \hat{\mathbf{g}}-t_{0} \mathbf{k}_{0} \cdot \hat{\mathbf{g}}=0+\left(\ell_{0}+p_{0}\right) \sin \left(\alpha_{R}+\alpha_{S}\right)$

$=359.0 \sin 11.85=73.7 \mathrm{~mm}$,

$\mathbf{q} \cdot \hat{\mathbf{n}}=-s_{0} \mathbf{h}_{0} \cdot \hat{\mathbf{n}}-t_{0} \mathbf{k}_{0} \cdot \hat{\mathbf{n}}=-\left(\ell_{s 0}+p_{S 0}\right)+\left(\ell_{0}+p_{0}\right)$

$\times \cos \left(\alpha_{R}+\alpha_{S}\right)=-304.0+351.3=47.3 \mathrm{~mm}$,

$a=t_{0} \mathbf{k}_{0} \cdot \hat{\mathbf{n}} /\left(s_{0} \mathbf{h}_{0} \cdot \hat{\mathbf{n}}\right)=-\left(\ell_{0}+p_{0}\right) \cos \left(\alpha_{R}+\alpha_{S}\right) /$

$\times\left(\ell_{S 0}+p_{S 0}\right)=-351.3 / 304.0=-1.156$

$\lambda=\hat{\lambda}\left(\ell_{S 0}+p_{S 0}\right) / \ell_{S 0}=(0.050)(304.0 / 73.97)$

$=0.206 \mathrm{~mm}$,

where all angles are written in deg. The two components $\Delta \mathbf{r} \cdot \hat{\mathbf{n}}$ and $\Delta \mathbf{r} \cdot \hat{\mathbf{g}}$ of the vector $\Delta \mathbf{r}=\tilde{x} \mathbf{e}_{1}+\tilde{y} \mathbf{e}_{2}$ give the variation of $\delta \tilde{D}_{M}$ across the field of view. With $\hat{\mathbf{n}}=\mathbf{e}_{1} \sin \alpha_{S}-\tilde{\mathbf{n}} \cos \alpha_{S}$ and $\hat{\mathbf{g}}=-\mathbf{e}_{1} \cos \alpha_{S}-\tilde{\mathbf{n}} \sin \alpha_{S}$, we find

$\Delta \mathbf{r} \cdot \hat{\mathbf{n}}=\tilde{x} \sin \alpha_{S}=\tilde{x} \sin 5.15$,

$\Delta \mathbf{r} \cdot \hat{\mathbf{g}}=-\tilde{x} \cos \alpha_{S}=-\tilde{x} \cos 5.15$.

Introducing Eqs. (36) and (40) in Eqs. (38) and (39), they become, respectively,

$$
\begin{aligned}
& \delta \tilde{D}_{M}=\left(A_{1}+\frac{A_{2} \tilde{x}}{1+A_{3} \tilde{x}}\right) \frac{\tilde{z}}{1+A_{3} \tilde{x}+A_{4} \tilde{z}+A_{5} \tilde{x} \tilde{z}}, \\
& \delta \tilde{D}_{M L}=\left(A_{1}+A_{2} \tilde{x}\right) \tilde{z},
\end{aligned}
$$

$A_{1}=\frac{\sin \left(\alpha_{R}+\alpha_{S}\right)}{\lambda \cos \alpha_{R}}=1.006 \mathrm{~mm}^{-1}$

$A_{2}=-\frac{(1+a) \cos \alpha_{S}}{\lambda\left(\ell_{0}+p_{0}\right) \cos \alpha_{R}}=2.117 .10^{-3} \mathrm{~mm}^{-2}$,

$A_{3}=\frac{\sin \alpha_{S}}{\ell_{S 0}+p_{S 0}}=2.953 .10^{-4} \mathrm{~mm}^{-1}$,

$A_{4}=-\frac{\cos \left(\alpha_{R}+\alpha_{S}\right)}{\left(\ell_{S 0}+p_{S 0}\right) \cos \alpha_{R}}=-3.242 .10^{-3} \mathrm{~mm}^{-1}$,

$A_{5}=-\frac{\sin \alpha_{S}}{\left(\ell_{0}+p_{0}\right)\left(\ell_{S 0}+p_{S 0}\right) \cos \alpha_{R}}=-8.281 .10^{-7} \mathrm{~mm}^{-2}$.
The experimental calibration consists in determining the fringe order change per unit length in depth of field for each pixel. Using a phase-shifting device in the projector enables measuring the changes in terms of a phase difference map. The frames were digitized with 8 -bit resolution, with one fringe order corresponding to a phase change of $2 \pi$ or 256 gray levels.

Figure 13 shows lines of equal moiré fringe order, i.e., equal phase, for a succession of 10 translation steps. Because the optic of the projector and that of the camera are not parallel, the density of moire fringes varies across the calibration plane. For a step of $\Delta \tilde{z}=1.00 \mathrm{~mm}$, the pattern repeats itself, indicating a shift of approximately one fringe order per millimeter. A meridian cross section (over the width) through the calibration planes of Fig. 13 shows the corresponding moiré surfaces intersecting the calibration plane in its different positions (Fig. 14). The phase differences were then obtained by digitally subtracting the phase map of the zero position from the others. The result is a difference in fringe order shown in the comparative phase difference map of Fig. 15, with the difference moire as function of the field of view in millimeters. Figure 15 simultaneously represents our

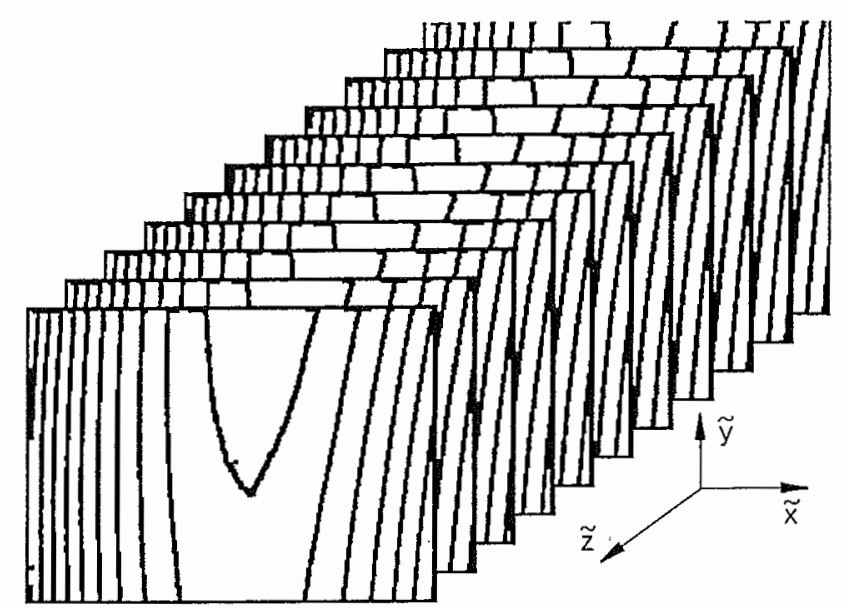

Fig. 13 Lines of equal moiré fringe order for a succession of 10 translation steps.

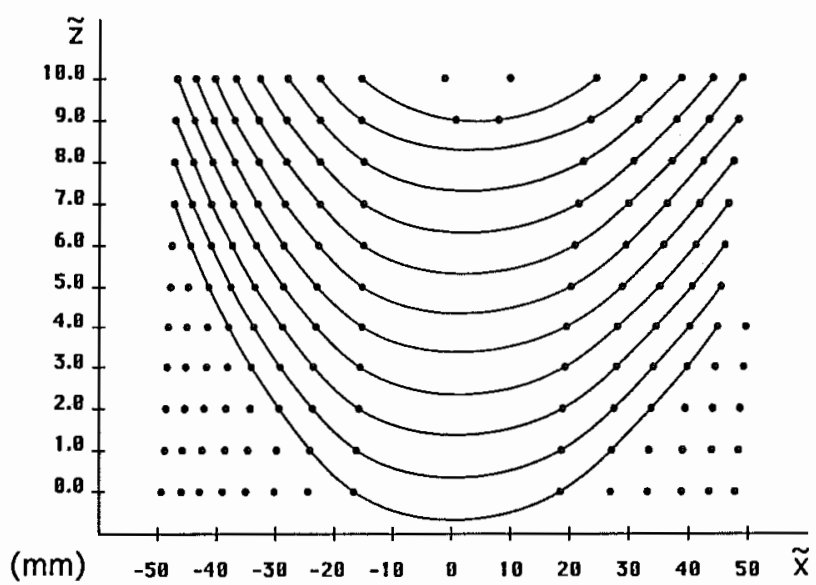

Fig. 14 Visualization of the moiré surfaces. 


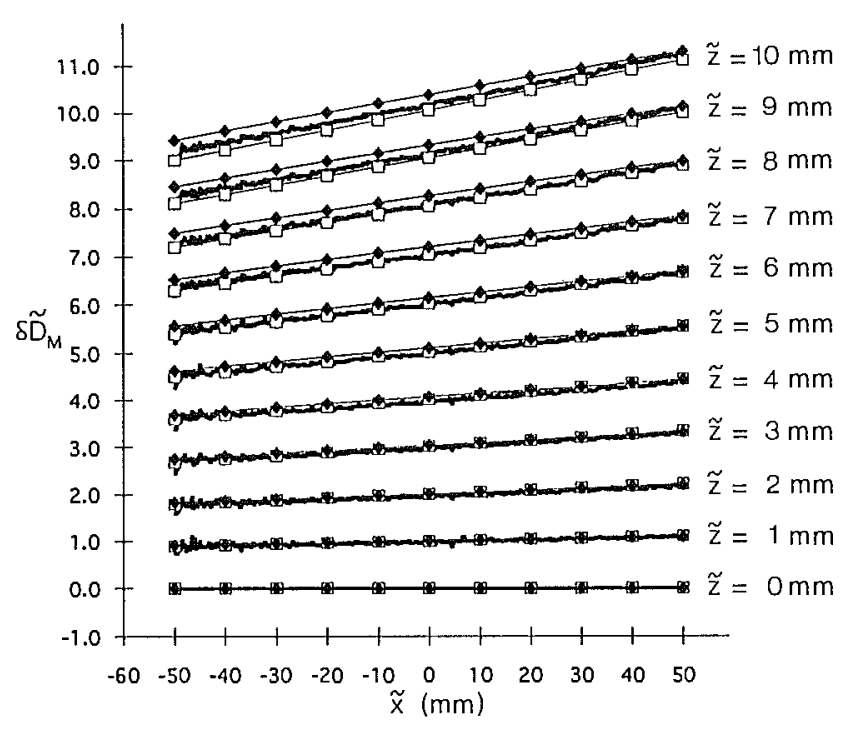

Fig. 15 Comparative phase difference map: -experimental, exact, and $\square$ linear.

experimentally measured difference moiré and that theoretically computed with Eqs. (41) and (42). This confirms that the experiment and the theory are in agreement. The experimental phase difference map contains the calibration factors over the width of the field of view for each translation step. Note that the difference moiré varies almost linearly over the width by approximately $20 \%$ change, whereas the change over the height is negligible. Note also that a small change of $\alpha_{R}$ and $\alpha_{S}$ first influences the value of $\delta \tilde{D}_{M}$, whereas a small change of $\ell_{0}+p_{0}$ and $\ell_{S 0}+p_{S 0}$ first influences the variation of $\delta \tilde{D}_{M}$ across the field of view.

To properly calibrate a projection moiré measurement, the relative moire $\Delta D_{M}$ corresponding to the calibration plane in its initial position must be subtracted from the relative moiré $\Delta \tilde{D}_{M S}$ corresponding to the object shape. The result is a difference moiré $\delta \tilde{D}_{M S}=\Delta \tilde{D}_{M S}-\Delta D_{M}$, which must be compared with our experimental phase difference map $\delta \tilde{D}_{M}$. If no experimental map is available, it is also possible to use in a first approximation a theoretically computed one if the angles and the lengths have been measured carefully.

In the linear case, for a fixed value $\tilde{x}=\tilde{x}_{0}$ across the field of view, which means for a fixed pixel of our CCD array, we can determine the constant proportionality factor $A=A_{1}+A_{2} \tilde{x}_{0}=\delta \tilde{D}_{M L} / \tilde{z}_{c}$ of Eq. (42), where $\tilde{z}_{c}$ is the known displacement of the calibration plane corresponding to the value of $\delta \tilde{D}_{M} \simeq \delta \tilde{D}_{M L}$ in our phase difference map. Because the difference moiré $\delta \tilde{D}_{M S}$ of the object shape obeys the same law, we can divide it by this constant factor to get approximately the displacement $\tilde{z}$ as follows:

$\tilde{z} \simeq \frac{\delta \tilde{D}_{M S}}{A}$, with $A=A_{1}+A_{2} \tilde{x}_{0}=\frac{\delta \tilde{D}_{M L}}{\tilde{z}_{c}} \simeq \frac{\delta \tilde{D}_{M}}{\tilde{z}_{c}}$.

In our experiment, this linear approximation is accurate enough. For example, Fig. 16 shows the surface shape under load of a carbon fiber reinforced polymer (CFRP) panel reinforced with stringers on the back side. The phase map is

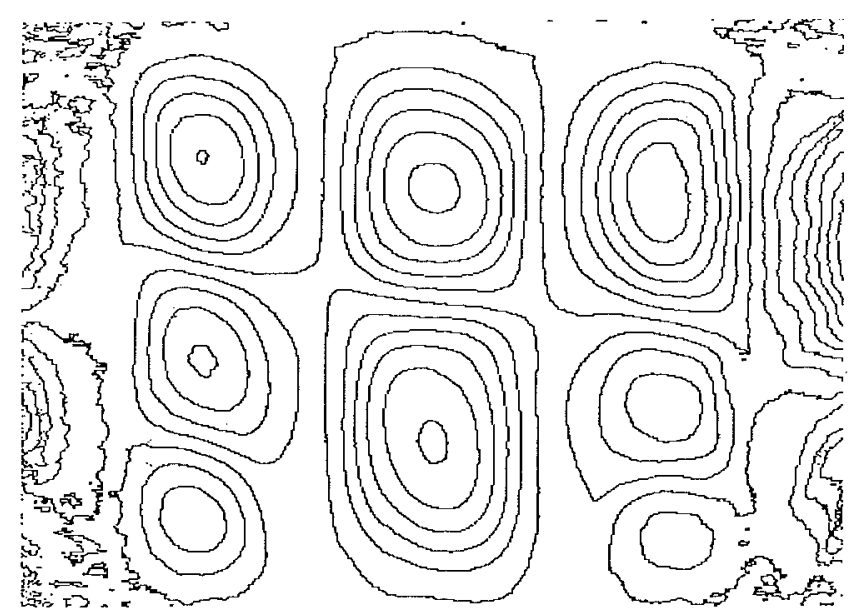

Fig. 16 Contour plot of a CFRP panel under load.

pseudocolor processed to show a contour plot display with $0.5-\mathrm{mm}$ displacement in depth of field between each level line.

In the exact case, the nonlinear problem of calibration can be solved in a similar way. For the same fixed value $\tilde{x}=\tilde{x}_{0}$ across the field of view, we write Eq. (41) as follows $(i=1$, $2,3, \ldots)$ :

$\tilde{z}_{c i}=\frac{B_{1} \delta \tilde{D}_{M i}}{1-B_{2} \delta \tilde{D}_{M i}}$, with $\quad B_{1}=\frac{1+A_{3} \tilde{x}_{0}}{A_{1}+\left(A_{2} \tilde{x}_{0} / 1+A_{3} \tilde{x}_{0}\right)} \quad$ and

$B_{2}=\frac{A_{4}+A_{5} \tilde{x}_{0}}{A_{1}+\left(A_{2} \tilde{x}_{0} / 1+A_{3} \tilde{x}_{0}\right)}$.

The constant factors $B_{1}$ and $B_{2}$ can be determined by taking at least two sets of values $\delta \tilde{D}_{M i}$ and $\tilde{z}_{c i}$ in our phase difference map. The exact displacement $\tilde{z}$ corresponding to the difference moiré $\delta \tilde{D}_{M S}$ is then given by the following expression:

$$
\begin{aligned}
& \tilde{z}=\frac{B_{1} \delta \tilde{D}_{M S}}{1-B_{2} \delta \tilde{D}_{M S}} \text {, with } B_{1}=\frac{\tilde{z}_{r 1} \tilde{z}_{c 2}\left(\delta \tilde{D}_{M 2}-\delta \tilde{D}_{M 1}\right)}{\left(\tilde{z}_{c 2}-\tilde{z}_{c 1}\right) \delta \tilde{D}_{M 1} \delta \tilde{D}_{M 2}} \text { and } \\
& B_{2}=\frac{\tilde{z}_{c 2} \delta \tilde{D}_{M 1}-\tilde{z}_{c 1} \delta \tilde{D}_{M 2}}{\left(\tilde{z}_{c 2}-\tilde{z}_{c 1}\right) \delta \tilde{D}_{M 1} \delta \tilde{D}_{M 2}} . \\
& 7.3 \quad \begin{array}{l}
\text { Special Case of Projection Moiré with Central } \\
\text { Projection }
\end{array}
\end{aligned}
$$

\subsection{Special Case of Projection Moiré with Central Projection}

This case concerns central projection and observation with parallel optical axes, the two grid planes being perpendicular to their respective optical axes. This particular case can be obtained from the general case [Eq. (19)] by assuming that $\overline{\mathbf{n}}=-\mathbf{k}_{m}=\mathbf{h}_{m}=\hat{\mathbf{n}}$ and $\ell_{0}=\ell_{S 0}, \ell_{0} /\left(\ell_{0}+p_{0}\right)=\ell_{S 0} /$ $\left(\ell_{S 0}+p_{S 0}\right)$. The two distances $L_{0}$ and $L_{S 0}$ are not necessarily the same, but they are small because of focusing near the object. In our model (Fig. 17), we have $\widehat{\mathbf{N}}=\mathbf{K}_{m}=\mathbf{H}_{m}=\hat{\mathbf{N}}$, $\widehat{\mathbf{g}} \equiv \mathbf{K}_{m} \overline{\mathbf{g}}, \quad \hat{\mathbf{g}} \equiv \mathbf{K}_{m} \hat{\mathbf{g}}, \quad \widehat{\mathbf{M}}_{m} \Delta \boldsymbol{\rho}=\hat{\mathbf{M}}_{m} \Delta \boldsymbol{\rho}=\Delta \boldsymbol{\rho}, \quad \widehat{\mathbf{M}}=\widehat{\mathbf{M}}_{m}, \quad \hat{\mathbf{M}}$ $=\hat{\mathbf{M}}_{m}=\mathbf{I}-\left[\mathbf{h} \otimes \mathbf{k}_{m} /\left(\mathbf{h} \cdot \mathbf{k}_{m}\right)\right]$, and Eq. (19) becomes 


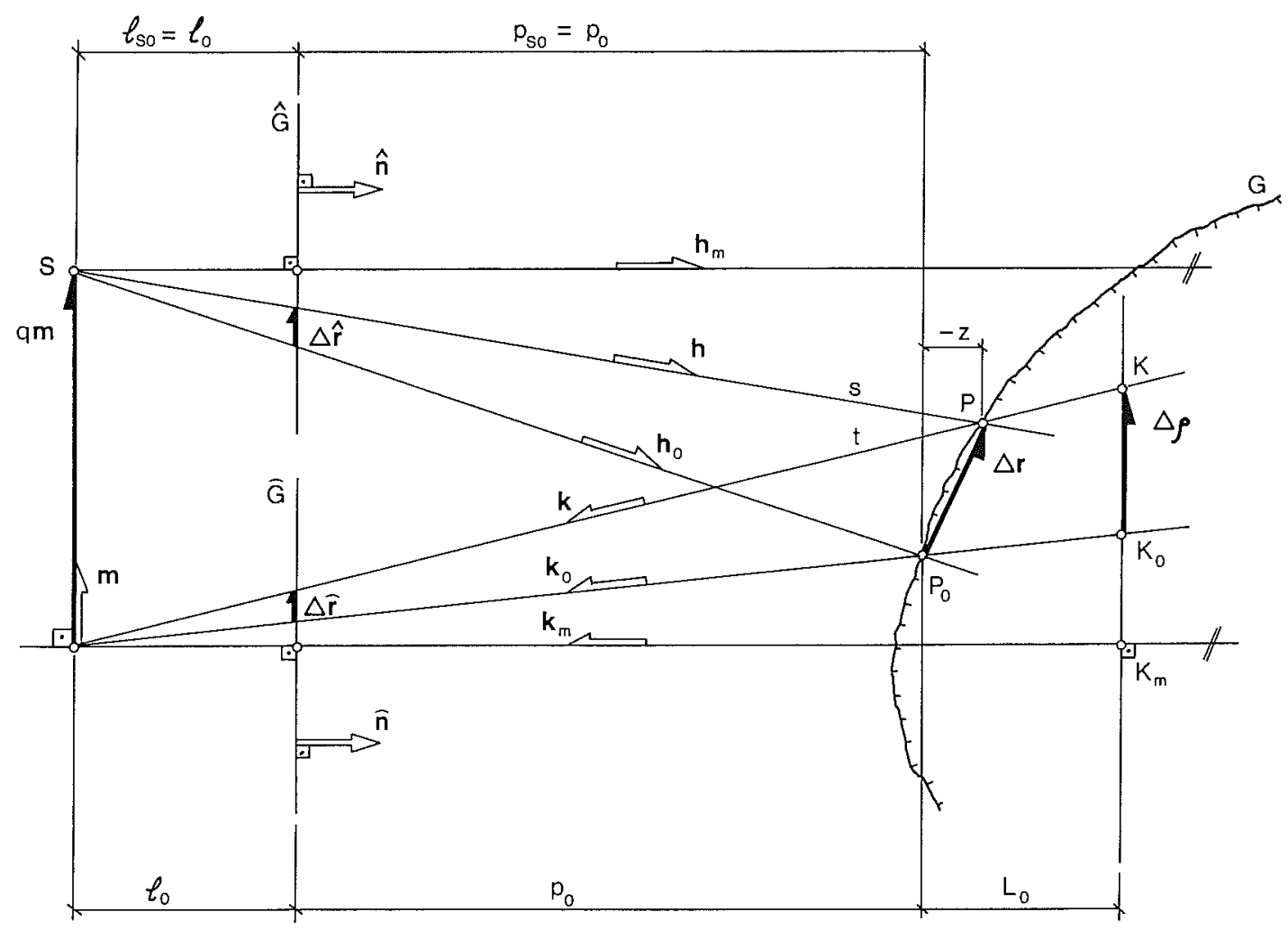

Fig. 17 Special case of projection moiré with central projection and parallel optical axes.

$$
\begin{aligned}
\Delta D_{M}= & \frac{\ell_{S 0}}{\ell_{S 0}+p_{S 0}}\left(\frac{1}{\lambda} \widehat{\mathbf{g}} \widehat{\mathbf{M}}_{m}-\frac{1}{\hat{\lambda}} \hat{\mathbf{g}} \hat{\mathbf{M}}_{m}\right) \cdot \Delta \mathbf{r}=\mathbf{p}(\mathbf{k}, \mathbf{h}) \cdot \Delta \mathbf{r} \\
= & \frac{\ell_{0}}{\ell_{0}+p_{0}+L_{0}}\left(\frac{1}{\hat{\lambda}} \widehat{\mathbf{g}}-\frac{1}{\hat{\lambda}} \hat{\mathbf{g}}\right) \cdot \mathbf{K}_{m} \Delta \mathbf{\rho} \\
& -\frac{\ell_{S 0}}{\hat{\lambda}\left(\ell_{S 0}+p_{S 0}\right)}\left(\frac{\hat{\mathbf{g}} \cdot \hat{\mathbf{M}}_{m b} \mathbf{k}}{\mathbf{k} \cdot \mathbf{k}_{m}}\right) z .
\end{aligned}
$$

Let us now consider the more special case, where $\overline{\mathbf{g}} / \widehat{\lambda}=$ $\hat{\mathrm{g}} / \hat{\lambda}$. Equation (48) becomes

$\Delta D_{M}=-\frac{z \ell_{S 0}}{\hat{\lambda}\left(\ell_{S 0}+p_{S 0}\right)}\left(\frac{\mathbf{k}}{\mathbf{k} \cdot \mathbf{k}_{m}}-\frac{\mathbf{h}}{\mathbf{h} \cdot \mathbf{k}_{m}}\right) \cdot \hat{\mathbf{g}}$.

To get the geometrical term in parenthesis, we write and solve the following vector equation system:

$$
\begin{aligned}
& \left.\begin{array}{l}
q \mathbf{m}+s \mathbf{h}+t \mathbf{k}=0 \\
t \mathbf{k}=\mathbf{K}_{m} \mathbf{x}_{k}+\left(\ell_{S 0}+p_{S 0}-z\right) \mathbf{k}_{m} \\
s \mathbf{h}=-\left(\ell_{S 0}+p_{S 0}-z\right) \mathbf{k}_{m}+\mathbf{K}_{m} \mathbf{x}_{h}
\end{array}\right\} \\
& \Rightarrow \frac{\mathbf{k}}{\mathbf{k} \cdot \mathbf{k}_{m}}-\frac{\mathbf{h}}{\mathbf{h} \cdot \mathbf{k}_{m}}=-\frac{q \mathbf{m}}{\ell_{S 0}+p_{S 0}-z},
\end{aligned}
$$

with $q \mathbf{m}=\mathbf{R S}, \mathbf{m} \cdot \mathbf{m}=1, t \mathbf{k}=\mathbf{P R}, s \mathbf{h}=\mathbf{S P}$, and where $\mathbf{K}_{m} \mathbf{x}_{k}$ and $\mathbf{K}_{m} \mathbf{x}_{h}$ are some unknown vectors in the plane normal to $\mathbf{k}_{m}$. As we can see, Eq. (50) is constant for a fixed $z$. With $\mathbf{m} \cdot \hat{\mathbf{g}}=\cos \varphi \neq 0$, Eq. (49) for $\Delta D_{M}$ describes pseudolevel lines respective to a plane normal to $\mathbf{k}_{m}$ passing through the point $P_{0}$. These pseudolevel lines are not equally spaced and are given by the following well-known hyperbolic function [Eq. (5) in Ref. 19]:

$$
z=\frac{\hat{\lambda} \Delta D_{M}}{\left(q \ell_{S 0} \cos \varphi\right) /\left[\left(\ell_{S 0}+p_{S 0}\right)^{2}\right]+\left(\hat{\lambda} \Delta D_{M}\right) /\left(\ell_{S 0}+p_{S 0}\right)} .
$$

\section{References}

1. J. L. Doty, "Projection moiré for remote contour analysis," $J . O p t$. Soc. Am. 73(3), 366-372 (1983).

2. B. Breuckmann, Bildverarbeitung und optische Messtechnik in der industriellen Praxis, Franzis-Verlag GmbH, München (1993).

3. D. M. Meadows, W. O. Johnson, and J. B. Allen, "Generation of surface contours by moiré patterns," Appl. Opt. 9(4), 942-947 (1970).

4. C. Chiang "Moiré topography," Appl. Opt. 14(1), 177-179 (1975).

5. L. Pirodda, "Shadow and projection moire techniques for absolute or relative mapping of surface shapes,"' Opt. Eng. 21(4), 640-649 (1982).

6. K. J. Gåsvik, Optical Metrology, John Wiley \& Sons, New York (1987).

7. K. J. Gåsvik and G. K. Robbersmyr, "Autocalibration of the setup for the projected moiré-fringe method," Exper. Tech. 17(2), 41 -44 (1993).

8. P. S. Theocaris, "Isopachic patterns by the moiré method," Exper Mech. 4, I53-159 (1964).

9. H. Takasaki, "Moiré topography," Appl. Opt. 9(6), 1467-1472 (1970).

10. H. Takasaki, "Moiré topography," Appl. Opt. 12(4), 845-850 (1973)

11. M. Idesawa, T. Yatagai, and T. Soma, "Scanning moiré method and automatic measurement of 3-D shapes," Appl. Opt. 16(8), 2152-2162 (1977).

12. S. Dubowski, K. Holly, A. L. Murray, and J. M. Wander, "Design optimization of moiré interferometers for rapid 3-D manufacturing inspection," Machine Vision Systems Integration in Industry, Proc. SPIE 1386, 10-20 (1990). 
13. O. Kafri and I. Glatt, The Physics of Moire Metrology, Wiley Series in Pure and Applied Optics, John Wiley \& Sons, New York (1990).

14. W. Schumann, J.-P. Zürcher, and D. Cuche, Holography and Deformation Analysis, Springer Series in Optical Sciences, Vol. 46, Springer Berlin Heidelberg (1985)

15. P. Tatasciore, "Récupération des franges d'interférence en interférométrie holographique appliquée aux grandes déformations des corps opaques," PhD Thesis No. 8917, Swiss Federal Institute of Technology, Zürich (1989)

16. B. Breuckmann and W. Thieme, "Computer-aided analysis of holographic interferograms using the phase-shift method," Appl. Opt. 24(14), 2145-2149(1985).

17. P. Tatasciore and H.-R. Meyer-Piening, "Measurement of Poisson's ratio for structural foam material by speckle interferometry," in Composites Testing and Standardisation, Proc. ECCM-CTS, European Conference on Composites Testing and Standardisation, P. J. Hogg, G.D. Sins, F. L. Matthews (BCS), A. R. Bunsell, and A. Massiah (EACM), Eds., pp. 521-530, Amsterdam (1992).

18. R. Srinivasan, C. S. Hartley, B. B. Raju, and J. Clave, "Measurement of neck development in tensile testing using projection moiré," Opt. Eng. 21(4), 655-662 (1982).

19. S. Oesch and L. Pflug, "Relevé en continu de la topographie fine des chaussées et des modèles hydrauliques par moiré de projection," Revue Française de Mécanique 2, 61-67 (1988).

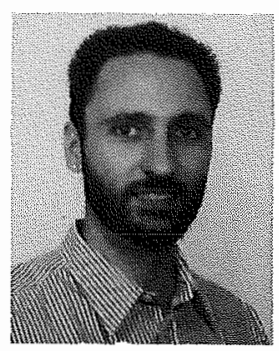

Philippe Tatasciore received his diploma in mechanical engineering in 1985 and his doctorate degree in technical sciences in 1989 from the Swiss Federal Institute of Technology in Zürich (ETHZ). During this time, he worked as research assistant at the Laboratory of Photoelasticity of the ETHZ. His primary interests are the study of large deformations with holographic interferometry, the nonlinear kinematic of deformation of curved surfaces, and the moiré metrology. Since 1991, he has been chief research assistant for optical measuring at the Institute of Lightweight Structures and Ropeways of the ETHZ.

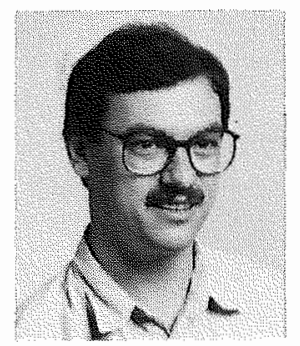

Erwin K. Hack received a diploma degree in theoretical physic's in 1986 and a PhD in physical chemistry in 1990 from the University of Zürich, Switzerland. In 1991 he joined the Electronics/Metrology Section of the Swiss Federal Laboratories for Materials Testing and Research (EMPA). He works on optical measurement techniques such as moiré and electronic speckle pattern correlation interferometry and their adaptation to various fields of materials testing. $\mathrm{He}$ is member of the SPIE working group on holography. 
